OPEN ACCESS

Edited by: Lucia Lopalco,

San Raffaele Hospital (IRCCS), Italy

Reviewed by:

Roberto Biassoni,

Giannina Gaslini Institute (IRCCS), Italy

Egle Kvedaraite,

Karolinska Institutet (KI), Sweden

*Correspondence:

Francisco Borrego

francisco.borregorabasco@

osakidetza.eus

†These authors have contributed equally to this work

Specialty section:

This article was submitted to

Viral Immunology,

a section of the journal

Frontiers in Immunology

Received: 19 January 2021 Accepted: 16 February 2021

Published: 11 March 2021

Citation:

Zenarruzabeitia O, Astarloa-Pando G,

Terrén I, Orrantia A, Pérez-Garay $R$,

Seijas-Betolaza I, Nieto-Arana J, Imaz-Ayo N, Pérez-Fernández S,

Arana-Arri E and Borrego F (2021) T Cell Activation, Highly Armed Cytotoxic Cells and a Shift in Monocytes CD300 Receptors Expression Is Characteristic of

Patients With Severe COVID-19.

Front. Immunol. 12:655934.

doi: 10.3389/fimmu.2021.655934

\section{T Cell Activation, Highly Armed Cytotoxic Cells and a Shift in Monocytes CD300 Receptors Expression Is Characteristic of Patients With Severe COVID-19}

\author{
Olatz Zenarruzabeitia ${ }^{1 \dagger}$, Gabirel Astarloa-Pando ${ }^{1 \dagger}$, Iñigo Terrén ${ }^{1}$, Ane Orrantia ${ }^{1}$, \\ Raquel Pérez-Garay ${ }^{1}$, Iratxe Seijas-Betolaza ${ }^{2}$, Javier Nieto-Arana ${ }^{3}$, Natale Imaz-Ayo ${ }^{4}$, \\ Silvia Pérez-Fernández ${ }^{4}$, Eunate Arana-Arri ${ }^{4}$ and Francisco Borrego ${ }^{1,5 *}$
}

\begin{abstract}
${ }^{1}$ Immunopathology Group, Biocruces Bizkaia Health Research Institute, Barakaldo, Spain, ${ }^{2}$ Intensive Care Medicine Service, Biocruces Bizkaia Health Research Institute, Cruces University Hospital, Barakaldo, Spain, ${ }^{3}$ Infectious Disease Service, Biocruces Bizkaia Health Research Institute, Cruces University Hospital, Barakaldo, Spain, ${ }^{4}$ Scientific Coordination Facility, Biocruces Bizkaia Health Research Institute, Barakaldo, Spain, ${ }^{5}$ Ikerbasque, Basque Foundation for Science, Billbao, Spain
\end{abstract}

COVID-19 manifests with a wide diversity of clinical phenotypes characterized by dysfunctional and exaggerated host immune responses. Many results have been described on the status of the immune system of patients infected with SARS-CoV-2, but there are still aspects that have not been fully characterized or understood. In this study, we have analyzed a cohort of patients with mild, moderate and severe disease. We performed flow cytometric studies and correlated the data with the clinical characteristics and clinical laboratory values of the patients. Both conventional and unsupervised data analyses concluded that patients with severe disease are characterized, among others, by a higher state of activation in all $\mathrm{T}$ cell subsets (CD4, CD8, double negative and $T$ follicular helper cells), higher expression of perforin and granzyme B in cytotoxic cells, expansion of adaptive NK cells and the accumulation of activated and immature dysfunctional monocytes which are identified by a low expression of HLA-DR and an intriguing shift in the expression pattern of CD300 receptors. More importantly, correlation analysis showed a strong association between the alterations in the immune cells and the clinical signs of severity. These results indicate that patients with severe COVID-19 have a broad perturbation of their immune system, and they will help to understand the immunopathogenesis of COVID-19.

Keywords: COVID-19, CD300a, CD300c, NK cells, T cells, monocytes, granzyme B, CD300e

\section{INTRODUCTION}

Severe acute respiratory syndrome coronavirus 2 (SARS-CoV-2) can cause coronavirus disease 2019 (COVID-19) which, in the worst cases scenario, can lead to severe manifestations such as acute respiratory distress syndrome, characterized by aggressive inflammatory responses in the lower part of respiratory tract, and multiple organ failure. A relevant number of symptomatic patients require hospitalization and a portion of them are admitted to the intensive care unit (ICU), moreover death 
may occur in a significant number of cases $(1,2)$. The thrombotic complications associated with COVID-19 represent a very important problem. Embolism and thrombosis are frequent clinical features of patients with severe COVID-19 $(3,4)$, sometimes despite anticoagulation therapy. Patients with severe disease have abnormal coagulation characteristics, including elevated D-dimer levels, and generalized thrombotic microvascular injury $(2,5,6)$.

In acute respiratory viral infections, pathology can be caused directly by the virus and/or by a damaging immune response from the host (7-9). In this sense, severe COVID-19 is due not only to the direct effects of SARS-CoV-2, but also to a misdirected host response with complex immune dysregulation $(10-15)$. Therefore, it is very important to exactly recognize and identify the immunological signatures that correlate with the severity of the disease, since this aspect undoubtedly has relevant clinical implications related to patients' stratification and management. From the first publications, the knowledge about the dysfunctional immune response in COVID-19 is constantly evolving. Most reports on immune dysfunction in COVID-19 patients have focused on severe disease. Hence, patients with severe COVID-19 exhibit in plasma higher amounts of numerous cytokines and chemokines than less severe cases (16-18). Severe manifestations are caused, in part, by high levels of interleukin (IL)-6 and the subsequent cytokine storm together with an altered type I interferon (IFN) response with low IFN production and an altered expression of IFN-regulated genes $(16,19)$. The cytokine storm is characterized by systemic inflammation, hemodynamic instability, hyperferritinemia and multiple organ failure (9). Many studies of circulating immune cells by flow and mass cytometry and/or single-cell RNA sequencing have provided valuable insights into immune perturbations in COVID-19 (10-14, 20-24). Recently, a multi-omics approach study has identified a major shift between mild and moderate disease, in which increased inflammatory signaling correlates with clinical metrics of blood clotting and plasma composition changes, suggesting that moderate disease may be the most effective situation for therapeutic intervention (14).

Lymphopenia, including $\mathrm{T}$ and NK cell lymphopenia, is a characteristic of severe COVID-19 (2, 10). In addition, alterations in the $\mathrm{T}$ cell compartment of COVID-19 patients have been described (11-13, 15). Among these, an increment in the frequency of activated and proliferating memory CD4 T cells and memory CD8 $\mathrm{T}$ cells in subsets of patients have been documented (11). Also, T cell exhaustion and increased expression of inhibitory receptors on peripheral $\mathrm{T}$ cells have been described $(11,25,26)$. Besides the evidences of $\mathrm{T}$ cell activation in COVID-19 patients, some studies have found decreases in polyfunctionality or cytotoxicity (26). Regarding to $B$ cells, it has been described an increase in the circulating plasmablasts and in proliferating $B$ cell subsets, among others (11). Alterations in natural killer (NK) cells during acute SARS$\mathrm{CoV}-2$ infection have also been reported. For example, reduced NK cell counts in patients with severe COVID-19 and impaired degranulating activity and IFN-gamma production in response to classical targets, such as K562 cells, have been published $(27,28)$. Other studies have shown that there is an increase in the frequency of NK cells displaying inhibitory receptors, such as NKG2A $(25,26,29)$. However, others have described a strong activation of both circulating and lung NK cells and an expansion of adaptive NK cells in patients with severe disease $(30,31)$.

Diverse immune mechanisms are on place to detect viral replication and protect the host. Pattern recognition receptors of the innate immune system recognize viral antigens and virusinduced damage, increasing bone marrow hematopoiesis, the release of myeloid cells including neutrophils and monocytes, and the secretion of cytokines and chemokines (32). If the inflammatory condition is not controlled, then emergency hematopoiesis may lead to bystander tissue damage that with the cytokine storm causes organ dysfunction. It is well-known that the myeloid compartment is also profoundly altered during SARS-CoV-2 infection, especially in patients with severe COVID-19 (23, 24, 33). Regarding circulating monocytes, alterations in the frequency of certain subpopulations have been described, such as intermediate $(\mathrm{CD} 14++\mathrm{CD} 16+)$ and non-classical $(\mathrm{CD} 14+\mathrm{CD} 16++)$ monocytes $(22-24,34,35)$. Some have described that loss of non-classical monocytes could help in the identification of high risk of severe COVID-19 $(24,34)$. Patients with severe disease are characterized by an accumulation of dysfunctional activated monocytes that express low levels of HLA-DR and immature neutrophils, indicating an emergency myelopoiesis, and an accumulation of these cells in the lungs $(23,24,36)$. Severe COVID-19 is also characterized by a profound alteration of neutrophil subsets. Neutrophilia with immature $\left(\mathrm{CD} 10^{\text {low }} \mathrm{CD} 101-\right)$ neutrophils, indicative of emergency granulopoiesis, and dysfunctional granulocytes are characteristics of patients with severe disease (24). A granulocytic signature has been proposed to identify SARS-CoV-2 infected from non-infected people as well as between severity stages (36). Severity was correlated with the expression of PD-L1 in granulocytes from patients with severe COVID-19 $(23,36)$.

Besides all the published data, there are still aspects not fully characterized in COVID-19 immunopathogenesis. Patients with severe disease exhibit a significant immune dysregulation and the nature of it is not completely understood. An in depth and complete knowledge of the dysregulated immune response is very important not only for its therapeutic implications, but also to better understand the immunopathology of the disease. Therefore, it is essential to entirely define the immune response characteristics related to disease features and determine at which stage of the disease specific therapeutic options may be most effective.

We have characterized lymphocytes and monocytes from patients with mild, moderate and severe disease using flow cytometry-based studies and correlated the results with clinical features and laboratory data. Comprehensive conventional and unsupervised analyses of the results showed that, in addition to others, the activation status of $\mathrm{T}$ lymphocytes and an increase in the cytotoxic potential of $\mathrm{T}$ and $\mathrm{NK}$ cells are correlated with the degree of the disease severity. Furthermore, we also describe an alteration in the expression of CD300 molecules in monocytes and granulocytes that, to our knowledge, was previously unrecognized. This alteration is characterized by a 
shift in the expression of this family of receptors between patients with moderate and severe COVID-19. Altogether, our results may help to further understand the immunopathogenesis of COVID-19.

\section{PATIENTS, MATERIALS, AND METHODS Patients and Healthy Donors}

In this study, we used plasma and whole blood samples cryopreserved in Cytodelics Stabilizer (http://www.cytodelics. com) from SARS-CoV-2 infected patients $(n=44)$ and adult healthy controls (HC) $(n=12)$. Patients were recruited at Cruces University Hospital. All samples were collected through the Basque Biobank for Research (https://www. biobancovasco.org). The Basque Biobank complies with the quality management, traceability and biosecurity, set out in the Spanish Law 14/2007 of Biomedical Research and in the Royal Decree 1716/2011. Patients recruited into the study tested PCR-positive for SARS-CoV-2, except one patient with mild disease, from March to June 2020 and were classified in 3 different clinical severity groups: mild, moderate and severe (see Supplementary Tables 1, 2). Patients were categorized using criteria from WHO "Clinical management of COVID-19." It was performed based on the following criteria: radiological signs of pneumonia, oxygen therapy needs during admission and oxygen saturation $\leq 90 \%$. HC were recruited among adults that did not have at any time history, nor signs or symptoms of COVID-19. All donors provided written and signed informed consent in accordance with the Declaration of Helsinki. This study was approved by the Basque Ethics Committee for Research with Medicines (CEIm-E) with the number CES-BIOEF 2020-13.

\section{Cell Preparation and Flow Cytometry}

From each donor a whole blood sample was collected in EDTA, from which one aliquot was centrifuged to obtain plasma and another aliquot was frozen using Cytodelics whole blood cell stabilizer. For extracellular staining, $200 \mu \mathrm{L}$ of blood + stabilizer mixture thawed at $37^{\circ} \mathrm{C}$ were incubated with the specific antibodies for $23 \mathrm{~min}$ at room temperature (RT) in the dark. Next, red blood cells were lysed using $2 \mathrm{~mL}$ of $1 \mathrm{X} B D$ FACS Lysing Solution (BD Biosciences) for $12 \mathrm{~min}$ at RT. Cells were washed with PBS containing $2.5 \%$ of bovine serum albumin (BSA) (Sigma-Aldrich) and were permeabilized and fixed using Cytofix/Cytoperm Plus Kit (BD Biosciences) following manufacturer's instructions. Then, intracellular staining was performed using the corresponding antibodies during $30 \mathrm{~min}$ at $4^{\circ} \mathrm{C}$ in the dark. Lastly, cells were washed, resuspended in $250 \mu \mathrm{L}$ of PBS and acquired in a LSRFortessa X-20 flow cytometer (BD Biosciences). Three flow cytometry panels were used to study $\mathrm{T}$ and $\mathrm{B}$ cells, NK cells and monocytes (see Supplementary Table 3).

\section{Flow Cytometry Data Analysis}

FCS 3.0 files were exported from the FACSDiva and imported into FlowJo v.10.7.1. for subsequent analysis. The following plug-ins were used: DownSample (1.1), t-distributed stochastic neighbor embedding (tSNE) and FlowSOM (2.6). Manual and automated analyses were performed. For the automated analysis, events were first downsampled from the gates of interest (CD4 T cells, CD8 T cells, B cells, monocytes, NK cells and CD56 ${ }^{\mathrm{dim}}$ NK cells) across all samples using DownSample plug-in. For each donor, CD4 T cells were downsampled to 1600 cells, CD8 T cells to 590 cells, B cells to 376 cells, monocytes to 5400 cells, NK cells to 557 cells and CD56 ${ }^{\mathrm{dim}}$ NK cells to 506 cells. Then, downsampled populations were concatenated for the analysis. FlowSOM was run using the indicated parameters in each figure.

\section{Clinical Laboratory Data}

Hemograms and serum determinations (D-dimer, ferritin, fibrinogen, CRP, etc.) from all patients were realized at the clinical laboratory of Cruces University Hospital. In addition, IL-6 levels and CMV serology were determined in plasma samples, also at the clinical laboratory of Cruces University Hospital. Samples for all determinations were obtained the same day than samples for flow cytometry analysis.

\section{Statistical Analysis and Data Representation}

GraphPad Prism v.8.4.3 was used for graphical representation and statistical analysis. Data were represented as boxplots with the median and 25-75th percentiles, and the whiskers denote lowest and highest values. Each dot represented a donor. Significance was determined by the Kruskal-Wallis test adjusting for multiple comparisons using Dunn's test. For categorical comparisons, the significance was determined by chi-squared test.

Correlation plots between variables were calculated and visualized as correlograms using R function corrplot. Spearman's Rank Correlation coefficient was indicated by square size and heat scale. Significance was indicated by ${ }^{*} p<0.05,{ }^{* *} p<0.01$, ${ }^{* * *} p<0.001$, and ${ }^{* * * *} p<0.0001$. $p$-values were adjusted using the Benjamini \& Hochberg test.

Bivariate analyses were performed (Supplementary Table 4). First, using the Shapiro-Wilks normality test we determined if variables followed a normal distribution. If they did, the average and standard deviation were reported, and if they did not have a normal distribution the median and interquartile range were indicated. To determine statistical significance, we use the Student's $t$-test if the variable follows a normal distribution and the Mann-Whitney $U$ test otherwise. To take into account multiple comparisons, we also presented the adjusted $p$-values using the Benjamini \& Hochberg test (Supplementary Table 4). Multivariate analysis was performed after selecting the statistically significant variables from bivariate analysis. To reduce the number of variables to include in the multivariate analysis we performed a Principal Component Analysis (PCA). Multinomial logistic regression models were performed to determine the components that were associated with the disease. 


\section{RESULTS}

\section{SARS-CoV-2 Infection: Study Design, Clinical Cohort, and Clinical Data}

Our aim was to evaluate the impact of acute SARS-CoV-2 infection in circulating leukocytes. To this end, we performed a cross-sectional study. Forty four patients with COVID-19 disease were recruited for the study. To correlate laboratory findings, including frequencies and phenotype of circulating leukocytes and the severity of the disease, we stratified our cohort of COVID-19 patients into 3 groups of those showing mild (15 patients), moderate (15 patients) and severe (14 patients) disease. The demographic, clinical characteristics and clinical laboratory values are summarized in Supplementary Tables 1, 2.

No significant differences were found between COVID-19 patients and $\mathrm{HC}$ in relation to age (median ages of 64 and 59.5 , respectively). There were also no significant differences between the three groups of patients (severe, moderate and mild) regarding the number of days from the appearance of symptoms and the sample collection: median of 8 days for the mild group (range: 0-35), 3 days for the moderate group (range: $0-15$ ) and 7 days for the severe group (range: 0-21). Regarding the gender of the participants, 7 (58.33\%) men and $5(41.66 \%)$ women participated in the HC group and 20 men (45.45\%) and 24 (54.54\%) women in the COVID-19 group (Figure 1A). As shown in Figure 1B, and in agreement with previous studies $(1,2,16,33)$, we observed an increase in the levels of plasma IL-6, C-reactive protein (CRP) and ferritin in COVID-19 patients in comparison with HC (Figure 1B). Specifically, $26 \%$ of patients exhibited IL-6 levels above the normal range $(>40 \mathrm{pg} / \mathrm{mL})$. Interestingly, all $\mathrm{HC}$ had IL-6 levels below the limit of detection $(<3 \mathrm{pg} / \mathrm{mL})$, while $69 \%$ of patients had $>3 \mathrm{pg} / \mathrm{mL}$ of IL-6. On the other hand, $66 \%$ of patients exhibited CRP levels above the normal range $(>11$ $\mathrm{mg} / \mathrm{L}$ ) and $57 \%$ of patients exhibited ferritin levels above the normal range ( $>300 \mathrm{ng} / \mathrm{mL}$ ) (Figure 1B). Furthermore, although white blood cell (WBC) counts were mostly normal in mild and moderate COVID-19 patients, some moderate and severe patients exhibited high WBC counts (Figure 1C). Also, and in accordance with the literature $(1,19)$, we observed frequencies and absolute numbers of lymphocytes below the normal values, and frequencies and absolute number of neutrophils above the normal values associated with the severity of the disease (Figure 1C). Finally, increased levels of IL-6 (>40 pg/mL), CRP (>11 mg/L), ferritin $(>300 \mathrm{ng} / \mathrm{mL})$, fibrinogen $(>400 \mathrm{mg} / \mathrm{dL})$ and D-dimer $(>500 \mathrm{ng} / \mathrm{mL})$ and lower levels of hemoglobin $(<13$ $\mathrm{g} / \mathrm{dL}$ ) were observed mostly in moderate and severe patients (Figure 1D).

To examine potential associations between these general laboratory values and other clinical features, we performed correlation analysis (Figure 1E). The analysis revealed associations between different degrees of severity with clinical features (oxygen therapy, bilateral infiltrations), comorbidities (hypertension), laboratory values (CRP, D-dimer, fibrinogen, hemoglobin), etc. Frequencies and absolute values of different subsets of WBCs were also correlated with severity degrees. Interestingly, the analysis did not reveal a correlation between
IL-6 levels with other parameters. Thus, COVID-19 patients presented varied and complex clinical phenotypes and laboratory values, including evidences of inflammation and altered leukocyte counts in many patients.

\section{SARS-CoV-2 Infection Is Associated With Activated CD4 T Cell Subsets Expressing Higher Levels of PD-1 and Perforin}

We next performed a detailed multiparametric flow cytometry analysis to further investigate circulating leukocytes status in COVID-19 patients (see gating strategy for each cell population in Supplementary Figure 1). Given the important role of T cells in the defense against viral infections and in the establishment of an immunological memory, as well as in the immunopathology and damage that may occur, we studied T cell subpopulations. We did not observe significant differences in the frequency of the major $\mathrm{T}$ cells subsets, i.e., CD4, CD8 and double negative $(\mathrm{DN})$ or in the $\mathrm{CD} 4 / \mathrm{CD} 8$ ratio between the patients and compared with the HC (Supplementary Figure 2). Four major CD4 T cell subpopulations were examined by using the combination of CD45RA and CD27 to define naïve (CD27+CD45RA+), memory (CD27+CD45RA-), effectormemory (CD27-CD45RA-), and terminal differentiated effectormemory (TEMRA) (CD27-CD45RA+) cells (Figure 2A). There were no significant differences in the frequencies of the four subsets between HC and COVID-19 patients.

Most viral infections induce proliferation and activation of $\mathrm{T}$ cells. The latter is detected by the coexpression of CD38 and HLA-DR (11). We found an expansion in the CD38+HLA-DR+ subset in all the non-naive CD4 T cell subsets from COVID19 patients, more significantly in the severe group (Figure 2B and Supplementary Figure 3A). Nevertheless, the magnitude of CD38+HLA-DR + cells expansion varied widely in our cohort and is significantly lower than the one observed in CD8 T cells (see below). After antigen recognition and activation, $\mathrm{T}$ cells up-regulate the expression of programed cell death-1 (PD1 ), with the aim of preventing an excessive response (37). Therefore, in the context of an acute infection, PD-1 could also be considered an activation marker, while during chronic stimulation, $\mathrm{T}$ cells became progressively dysfunctional and exhausted, and the expression of $\mathrm{PD}-1$ persists (37). We studied the expression of PD-1 on CD4 T cells and found that there was an increase in all non-naïve CD4 T cells from patients, which was statistically significant in the effector-memory subset from moderate and severe COVID-19 patients (Figure 2C and Supplementary Figure 3B). Similar to the CD38+HLA-DR+ cells, the expansion of PD-1+ cells varied widely, but importantly a strong positive correlation between CD38+HLA-DR + cells and PD-1+ cells was observed in COVID-19 patients (Figure 2D), suggesting the possibility that PD-1 expression on CD4 T cells during acute SARS-CoV-2 infection is more an activation marker than an exhaustion marker.

Given the relevant role of antibodies in the response to SARS-CoV-2, we also analyzed the circulating $\mathrm{T}$ follicular helper (TFH) cells (PD-1+CXCR5+) (38). We did not observe a significant increase, except for the moderate 
A
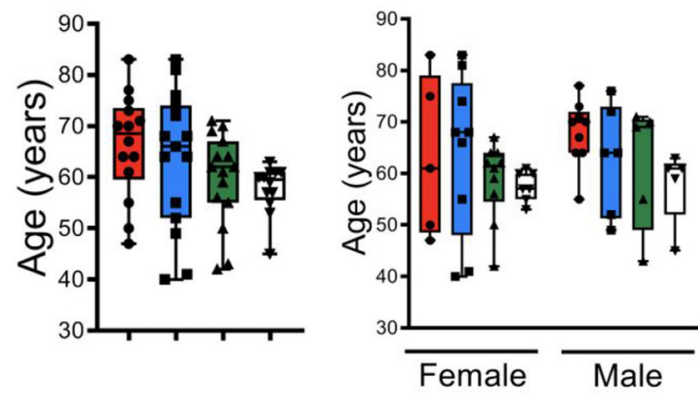

$\square$ Severe

$\square$ Moderate

$\square$ Mild

$\square \mathrm{HC}$
B

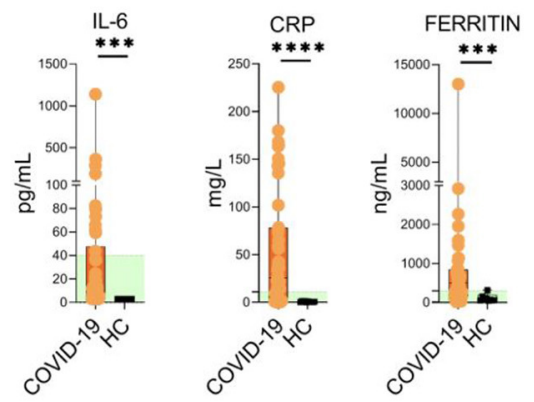

D
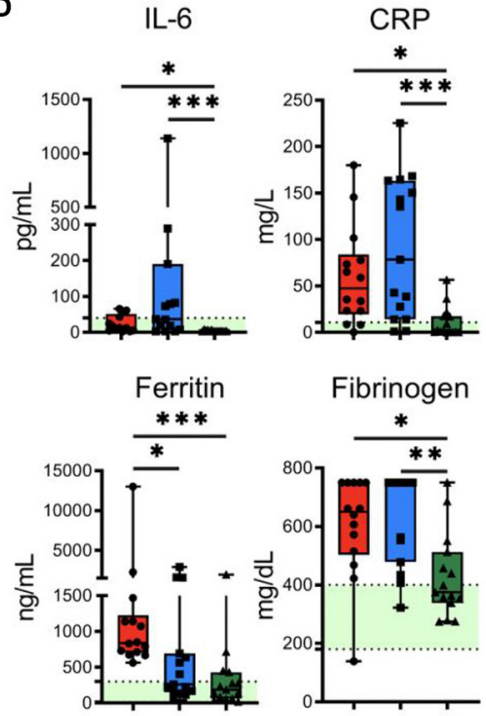

Fibrinogen

D-dime
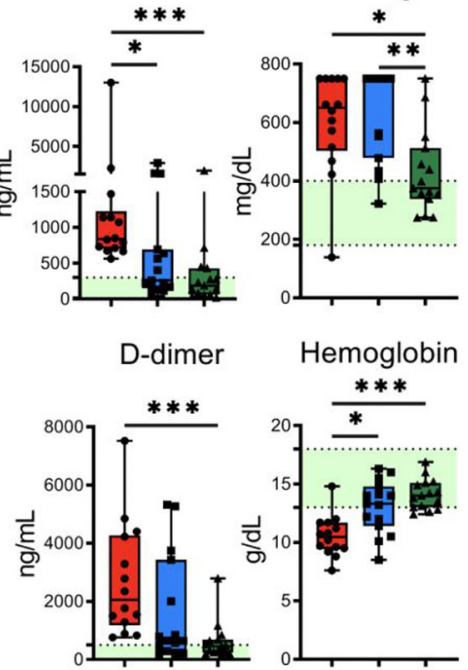

C

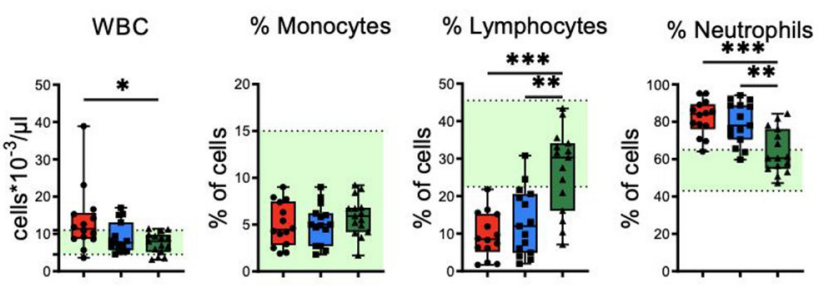

Monocyte count Lymphocyte count Neutrophil count

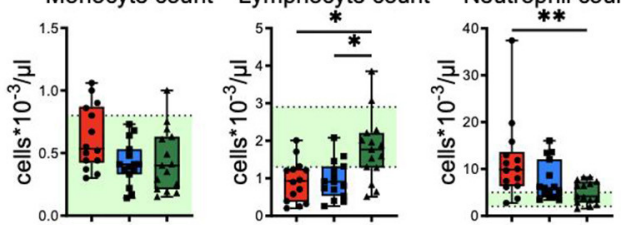

E

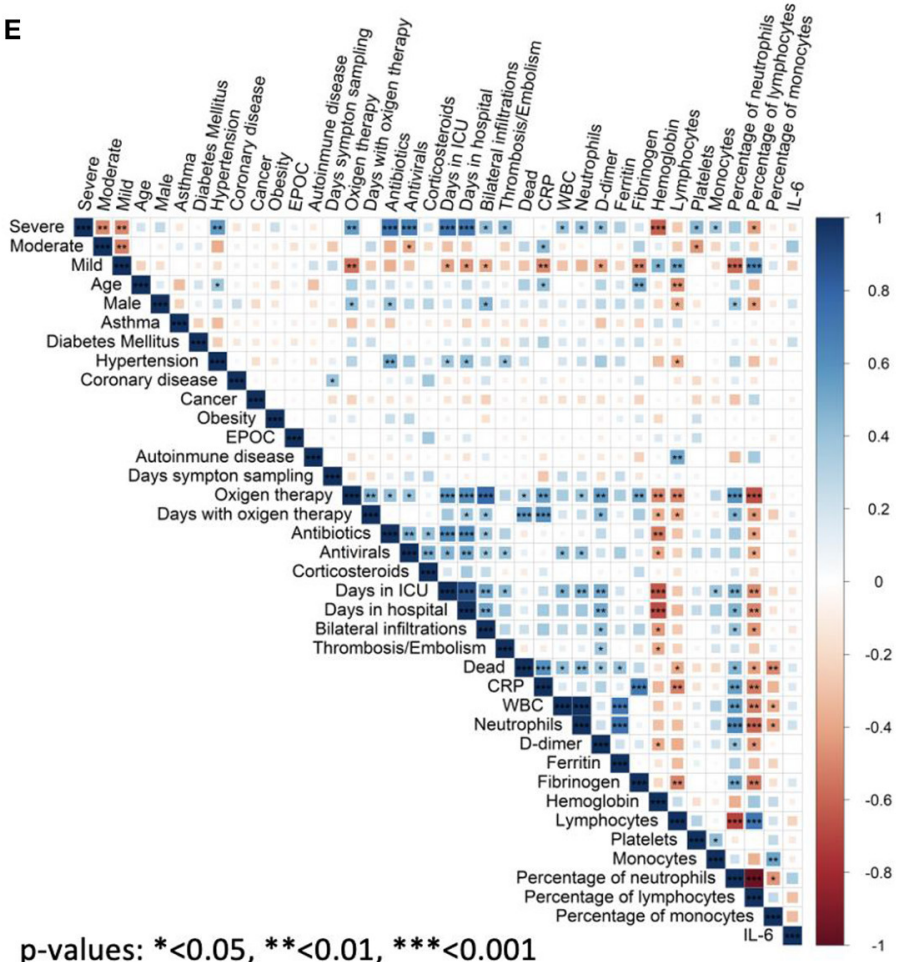

FIGURE 1 | Clinical features of patients, quantification of leukocyte subsets and inflammation markers. (A) Left: age and gender distribution of patient cohorts in this study, including healthy controls $(\mathrm{HC})$ and patients with mild (green), moderate (blue) and severe (red) COVID-19. Right: days from symptom onset to sample 
FIGURE 1 | collection. (B) Plasma levels of IL-6, C reactive protein (CRP) and ferritin in HC and COVID-19 patients. The ranges of normal clinical laboratory values are represented in light green. (C) White blood cells (WBC) counts, leukocyte subsets frequencies and counts in patients with mild, moderate and severe COVID-19. The light green region represents the normal range for healthy people in the clinical laboratory. (D) Plasma levels of IL-6, CRP, ferritin, fibrinogen, D-dimer and hemoglobin in COVID-19 patients. Normal clinical laboratory values are represented in light green. (E) Correlogram showing Spearman correlation of the indicated clinical features for COVID-19 patients. Data in (A,C,D) are represented as boxplot graphs with the median and 25-75th percentiles, and the whiskers denote lowest and highest values. Each dot represents a donor. Significance was determined by the Kruskal-Wallis test followed by Dunn's multiple comparison test. ${ }^{\star} p<0.05,{ }^{\star \star} p<0.01$, and ${ }^{\star * *} p<0.001$.

group of patients, in the frequency of TFH in COVID19 patients (Supplementary Figure 3C). Nevertheless, the frequency of CD38+HLA-DR+ TFH cells was expanded in patients, suggesting that they had a recent antigen encounter and have emigrated from the germinal center (38) (Supplementary Figure 3C). In addition, we performed an analysis of B cells and, while the frequencies of CD27$\mathrm{B}$ cells, which include mostly the naïve subset, tended to increase in COVID-19 patients, the frequencies of CD27+ memory B cells tended to decrease with the disease severity, although not significantly (Supplementary Figure 4A). Contrarily, the frequency of plasmablasts (CD27+CD38+) tended to increase, mostly in patients with moderate disease (Supplementary Figure 4A). We also observed a significant decrease in CXCR5 (Supplementary Figure 4B) and HLA-DR (Supplementary Figure 4C) expression levels in B cells from COVID-19 patients.

Cytotoxic CD4 T cells represent an additional mechanism by which CD4 $\mathrm{T}$ cells contribute to immunity. In viral infections, these perforin expressing CD4 T cells have been shown to play a protective and/or pathogenic role $(39,40)$. Therefore, we measured the expression of perforin in CD4 T cells from COVID19 patients (Figure 2E and Supplementary Figure 3D). Results showed that the frequency of effector-memory and TEMRA CD4 T cells expressing perforin from a subset of COVID-19 patients was higher than in HC (Figure 2E). Although the increase in the frequency of the memory cells that express perforin was not statistically significant between COVID19 patients and HC (Figure 2E), we observed an enhanced perforin expression per cell basis as shown by an increase in the median fluorescence intensity (MFI) of perforin + cells (Supplementary Figure 3D). Altogether, these results suggest that cytotoxic CD4 T cells may contribute to the clinical course of those patients.

To gain more insight, further define and also quantify these differences in a unsupervised manner, we performed FlowSOM clustering in non-naïve CD4 T cells and compared the expression of seven markers to define 12 clusters or populations (or Pop) (Figure 2F). Using this approach, we identified several populations differentially expressed between HC and COVID-19 patients (Figure 2G and Supplementary Figure 3E). For example, Pop10, which identified TEMRA cells (CD27CD45RA+) expressing perforin, was expanded in patients. Memory cells (CD27+CD45RA-) that express PD-1 and CD38 (Pop0) and PD-1, CD38 and HLA-DR (Pop2), as well as CD27-CD45RA ${ }^{\text {low }}$ expressing PD-1, CD38 and HLA-DR (Pop6) were also expanded in COVID-19 patients (Figure 2G). Thus, COVID-19 patients were characterized by expanded populations of activated, PD-1 and perforin expressing CD4 T cells in a subgroup of patients.

\section{SARS-CoV-2 Acute Infection Is Associated With CD8 T Cell Activation in Severe Patients}

CD8 T cells have a very relevant role in viral infections through their ability to recognize and kill virus infected cells and in the formation of the immunological memory. But also, highly differentiated CD8 T cells have been suggested to induce damage in SARS-CoV-2 infected lungs in an antigen-independent manner (41). Therefore, we next examined the four major subpopulations (naïve, memory, effector-memory and TEMRA). We observed no significant differences in the frequencies of the four subsets between HC and COVID-19 patients. Nevertheless, the frequency of CD8 effector-memory cells was significantly higher in patients with severe disease (Figure 3A).

Then, we determined the activation status of CD8 T cells. We observed that COVID-19 patients exhibited a significant expansion of activated (CD38+HLA-DR+) cells, especially in the patients with severe disease (Supplementary Figure 5A). When we looked at the CD8 T cells subsets, increased frequencies of activated CD8 $\mathrm{T}$ cells were also observed in patients, most significantly in those with severe disease (Figure 3B). The magnitude of the activated cells expansion varied widely, although in a significant subset of patients with severe COVID-19 more than $50 \%$ of their memory, effector-memory and TEMRA CD8 $\mathrm{T}$ cells were activated compared to $<10 \%$ in all $\mathrm{HC}$ (Figure 3B). We also studied the expression of PD-1 on CD8 T cells (Figure 3C and Supplementary Figure 5B) and although there was a tendency, no significant differences were observed between $\mathrm{HC}$ and patients, with the exception of an expansion of PD-1+ CD8 memory cells in patients with severe disease. In contrast to the CD4 T cells, we did not observe a correlation between CD38+HLA-DR+ cells and PD-1+ cells in COVID-19 patients (Supplementary Figure 5C), probably suggesting that PD-1 expression on CD8 T cells is more a marker of exhaustion than of activation. Nevertheless, more studies are required to confirm this statement.

CD8 T cells exert their cytotoxic activity after encountering virus-infected cells by releasing perforin and granzymes that are contained in their lytic granules (42). We next determine perforin expression in our cohort of COVID-19 patients. When we looked at the total CD8 $\mathrm{T}$ cell population we observed a significant increase in the frequency of cells containing perforin in patients with severe disease (Supplementary Figure 5D). This could be explained by an increase in the frequency of memory and effector-memory cells expressing perforin in severe patients 
A
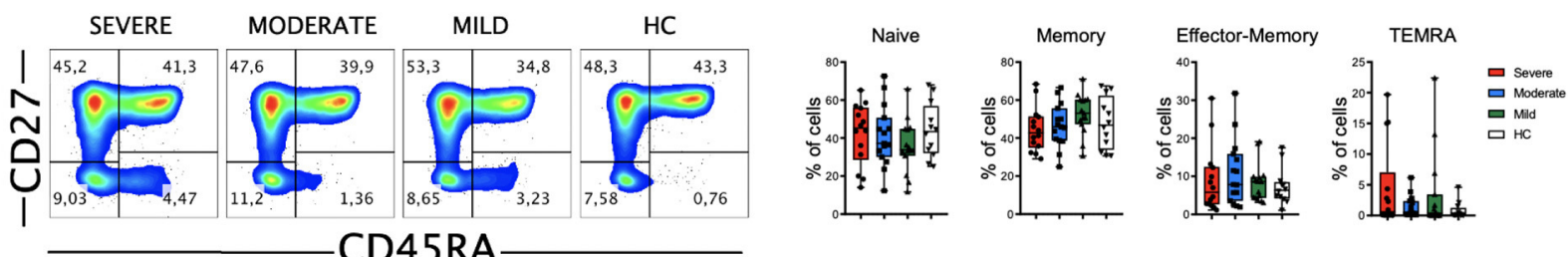

B
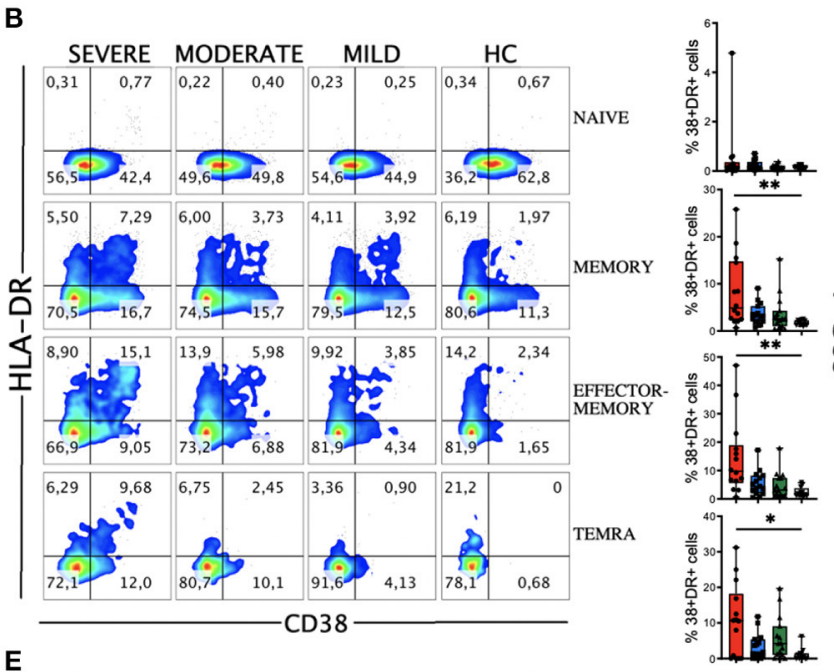

E

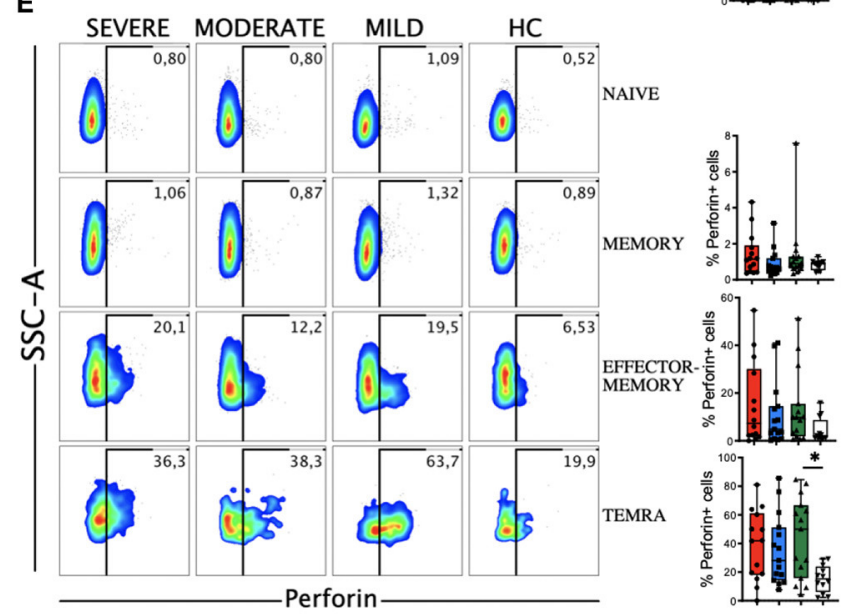

C
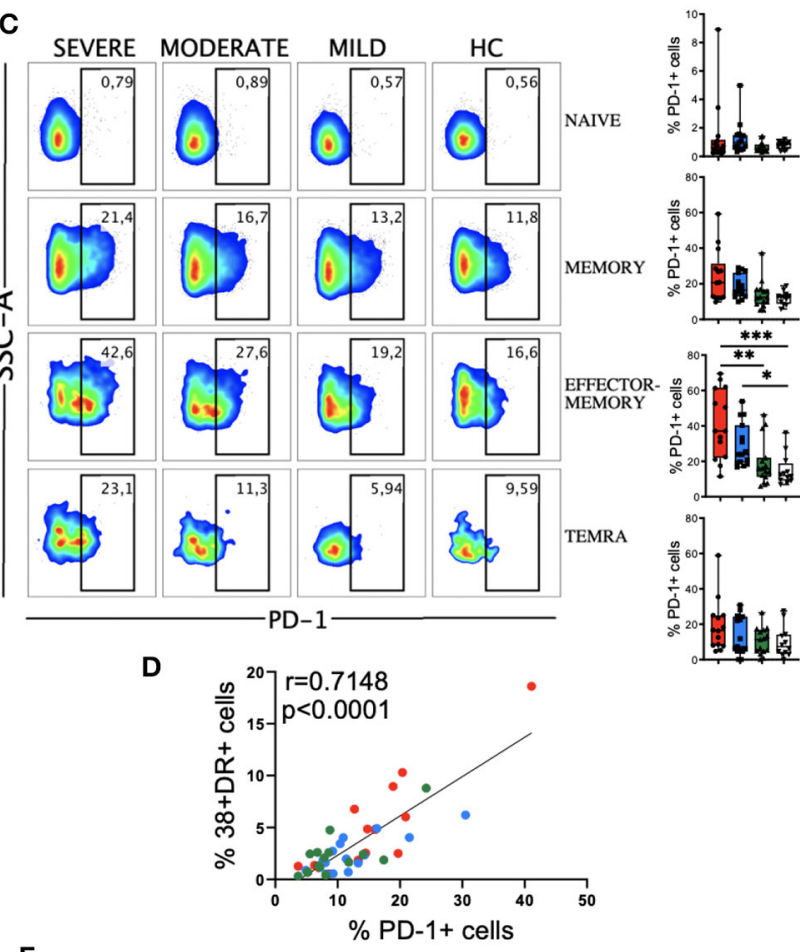

G
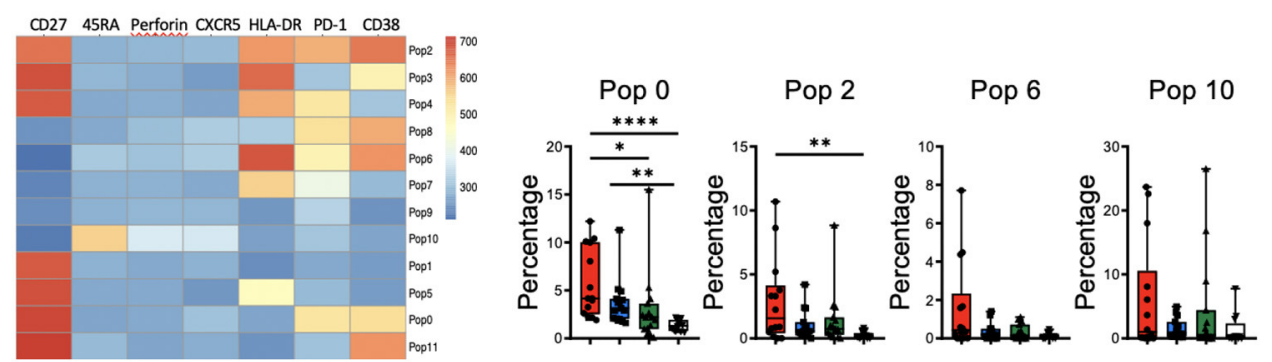

FIGURE 2 | CD4 T cell subsets, activation status and perforin expression in COVID-19 patients. (A) Left: pseudocolor plots of concatenated peripheral CD4 T cells from healthy controls $(\mathrm{HC})$ and patients with mild, moderate and severe disease. Four cell subsets were identified: naïve $(C D 27+C D 45 R A+)$, memory (CD27+CD45RA-), effector-memory (CD27-CD45RA-), and terminal differentiated effector-memory (TEMRA) (CD27-CD45RA+). Numbers in the quadrants are the average of each subset. Right: boxplot graphs representation of the data. (B) Pseudocolor plots of concatenated peripheral CD4T cells from HC and COVID-19 
FIGURE 2 | patients and boxplot graphs of the frequencies of activated naïve, memory, effector-memory and TEMRA cells. Numbers in the quadrants are the average of each subset. Activated T cells are identified by the coexpression of CD38 and HLA-DR. (C) Pseudocolor plots of concatenated peripheral CD4 T cells and boxplot graphs showing the frequencies of PD-1+ naïve, memory, effector-memory and TEMRA cells. Numbers in the gates are the average of PD-1+ cells in each subset. (D) Spearman correlation of activated (CD38+HLA-DR+) with PD-1+ CD4 T cells from patients with mild, moderate and severe COVID-19. (E) Pseudocolor plots of concatenated peripheral CD4 T cells and boxplot graphs of the frequencies of perforin positive naïve, memory, effector-memory and TEMRA cells. Numbers in the gates are the average of perforin positive cells in each subset. (F) tSNE projection of non-naïve CD4 T cell populations (Pop) identified by FlowSOM clustering tool. (G) Fluorescence intensity of each Pop as indicated in the column-scaled z-score and boxplot graphs showing the frequencies of Pop0, Pop2, Pop6, and Pop10 in HC and COVID-19 patients. Boxplots show the median and 25-75th percentiles, and the whiskers denote lowest and highest values. Each dot represents a donor. Significance of data in (A-C,E,H) was determined by the Kruskal-Wallis test followed by Dunn's multiple comparison test. ${ }^{*} p<0.05$, ${ }^{* *} p<0.01$, ${ }^{* * *} p<0.001$, and ${ }^{* * \star \star} p<0.0001$

(Figure 3D). Altogether, these results suggest that an expansion of activated and perforin containing non-naive CD8 T cells may contribute to the severity of the COVID-19 disease.

To gain more insight into the CD8 T cell alterations, we again used the FlowSOM clustering tool and compared the expression of several markers to define 12 populations (or Pop) within nonnaive CD8 + T cells (Figure 3E). We were able to identify some populations that were differentially expressed between COVID19 patients and HC (Figure 3F and Supplementary Figure 5E). The populations containing the activated cells (Pops 7, 8, 9, 10 , and 11) were significantly expanded in patients, especially in those with a severe disease. Pop7 identified activated memory CD8 T cells that are PD-1+, while Pop10 represented activated memory CD8 T cells that are PD-1-. Pop8 and Pop9 identified activated effector-memory CD8 T cells that are PD1 - and PD-1+, respectively (Figure 3F). Pop11 identified the activated TEMRA cell subset, which was significantly increased in severe patients, while the frequency of Pop0, which identified the TEMRA non-activated cells, was the same in $\mathrm{HC}$ and COVID-19 patients (Figure 3F and Supplementary Figure 5E). Pop1 identified the non-activated (CD38-HLA-DR-) and PD-1memory subset that decreased with the severity of the disease (Supplementary Figure 5E). Hence, COVID-19 patients were characterized by an expansion of non-naïve activated CD8 T cells, including both PD-1+ and PD-1- cells.

We have also analyzed the DN T cells, and in a similar way to what happens with $\mathrm{CD} 4$ and $\mathrm{CD} 8 \mathrm{~T}$ cells, the activated (HLA-DR+CD38+) and perforin expressing $\mathrm{DN}$ $\mathrm{T}$ cells are significantly expanded in COVID-19 patients (Supplementary Figure 6).

\section{Activated Monocytes, Decreased Levels of HLA-DR and a Shift in CD300 Receptors Expression Pattern Correlate With Severe COVID-19}

The myeloid cell compartment is profoundly dysregulated in patients with severe COVID-19 (22-24, 33, 36). In our cohort, we did not observe significant differences in the three main monocyte subsets, with the exception of intermediate monocytes in patients with moderate disease (Figure 4A). CD163 is a receptor expressed on monocytes that has been investigated as a potential inflammation marker in different infectious diseases (43). We found a significant increase in the percentage of CD163+ monocytes in patients with moderate and severe disease (Figure 4B). This increased frequency was observed in all the monocyte subsets, with a significant number of patients with moderate and severe disease exhibiting more than $40 \%$ of CD163 + intermediate monocytes (Figure 4B). Also, as previously published by others $(10,23,24)$, we found a gradual decrease in HLA-DR expression levels in all monocyte subsets that correlated with the severity of the disease (Figure 4C).

The CD300 molecules are type I transmembrane proteins expressed on the surface of immune cells that modulate a multitude of signaling pathways and have been found to be involved in several diseases, including viral infections and sepsis (44-47). Therefore, we decided to determine the expression of the inhibitory receptor $\mathrm{CD} 300 \mathrm{a}$ and activating receptors CD300c and CD300e on monocytes from patients with COVID19 (Figure 4D). Results showed a differential expression of these receptors between $\mathrm{HC}$ and patients. Very interestingly, we observed a shift in the expression of this family of receptors between moderate and severe disease. Specifically, while the expression levels of CD300a and CD300e increased in monocytes from patients with mild and moderate disease, a drastic decrease was observed in patients with a severe form of COVID-19. This decrease in the expression levels reached similar or lower levels than those expressed by patients with mild disease and HC (Figure 4D). The expression of CD300a in granulocytes $(\mathrm{CD} 66 \mathrm{~b}+$ cells $)$ was similar to monocytes, with an increase in patients with mild and moderate disease and then a decline in patients with severe COVID-19 (Supplementary Figure 7). The expression of CD300c on monocytes exhibited a somewhat opposite pattern to that of CD300a and CD300e (Figure 4D). In fact, the expression of this marker decreased in all patients, and significantly in those with a moderate disease. Although the mechanism responsible for the altered expression levels of CD300 molecules in patients with COVID-19 it is not known, these results, along with other observations, may be of great importance for distinguishing those patients who have a severe disease from others who have a moderate or mild illness.

Then, clustering was performed using FlowSOM and 10 populations (Pop) were identified and quantified (Figure 5A). Several populations were differentially expressed between HC and COVID-19 patients (Figure 5B and Supplementary Figure 8). For example, the classical monocytes Pop0 and Pop4, with low expression of HLA-DR, CD300a and $\mathrm{CD} 300 \mathrm{e}$, were significantly expanded in patients with severe disease. Interestingly, Pop0 expressed high levels of the CD300c and Pop4 exhibited low levels of this receptor. 
A
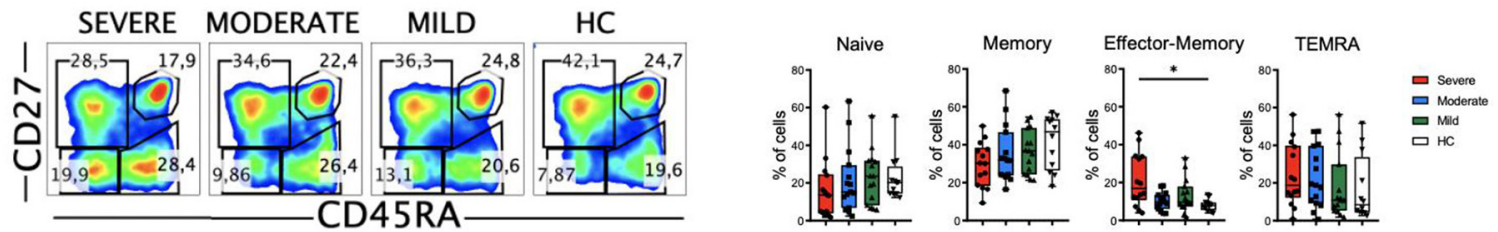

B

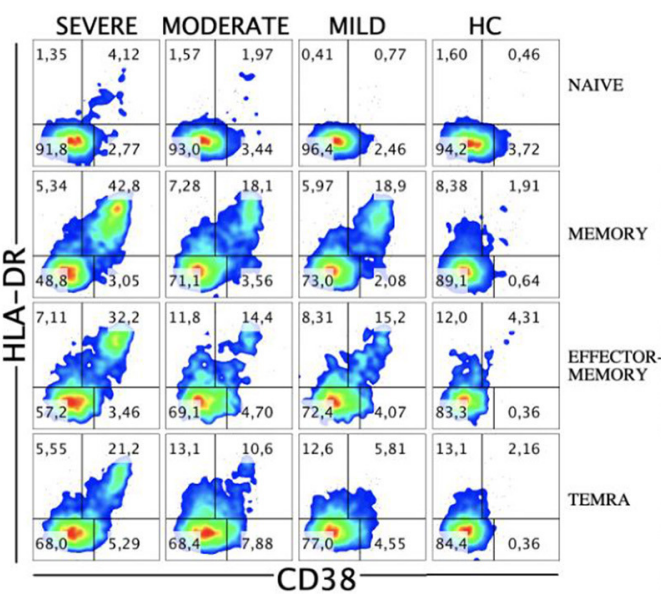

D

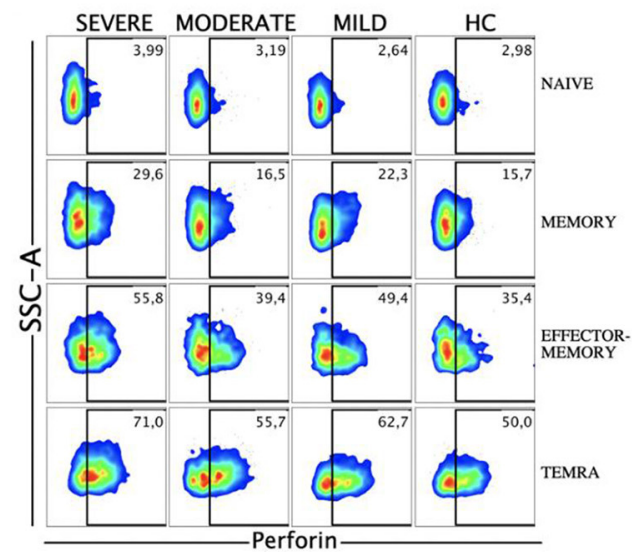

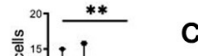

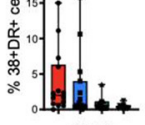

$\frac{200}{* * * *}$

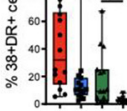

${ }^{100} \frac{* * * *}{\overline{8}}$

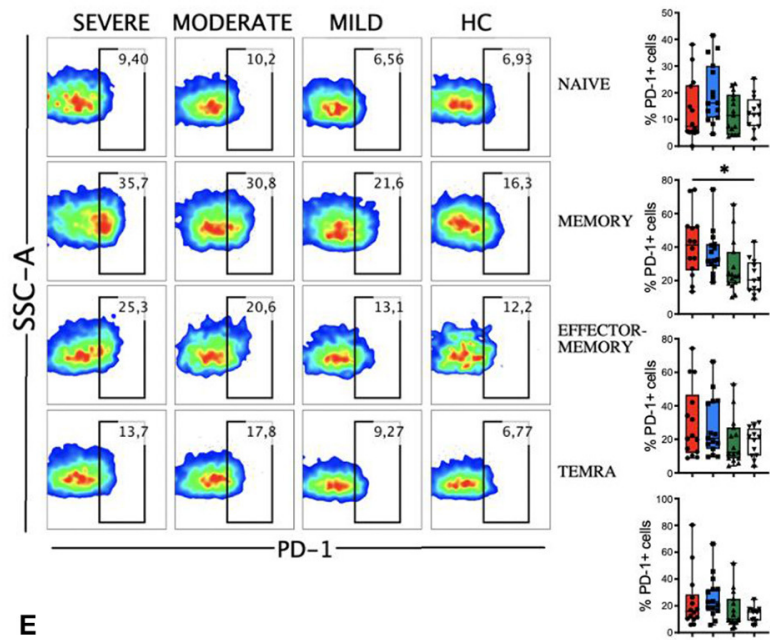

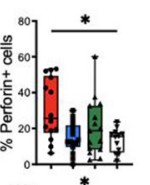
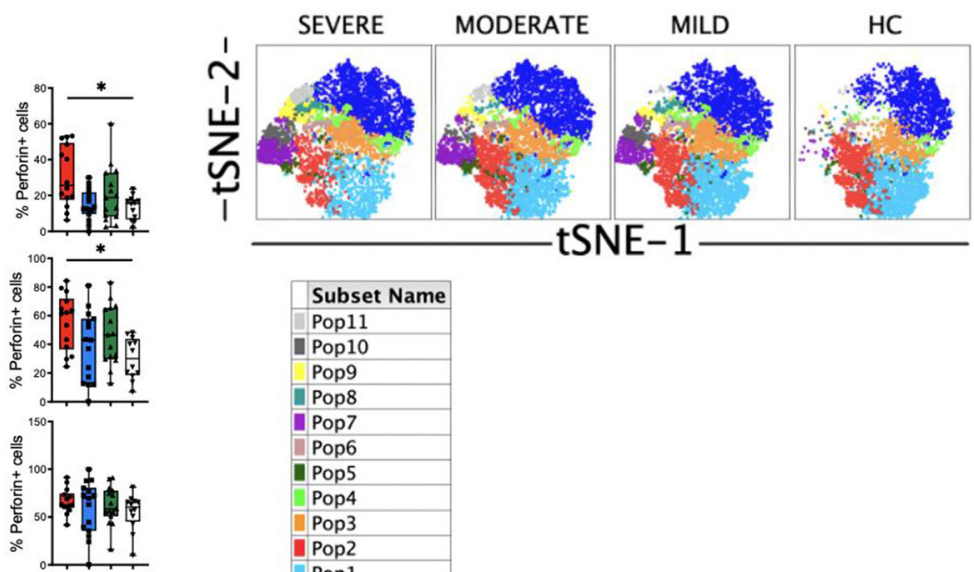

Subset Name

Pop11

\begin{tabular}{|l|l|} 
Pop10 \\
Pop9
\end{tabular}

\begin{tabular}{|l} 
Pop9 \\
\hline Pop8
\end{tabular}

Pop7

Pop6

$\begin{array}{r}\text { Pop5 } \\ \text { Pop4 } \\ \hline\end{array}$

\begin{tabular}{|l|l|}
\hline Pop4 \\
\hline Pop3 \\
\hline
\end{tabular}

\begin{tabular}{ll} 
Pop2 \\
\hline Pop1
\end{tabular}

Pop1
Pop0

CD27 HLA-DR CD38 45RA PD-1 Perforin CXCR5

$$
\begin{array}{l|l|}
\hline \text { Pop5 } & 700 \\
\hline \text { Pop1 } & 600 \\
\hline \text { Pop2 } & 500 \\
\hline \text { Pop0 } & 400 \\
\hline \text { Pop6 } & 300 \\
\hline \text { Pop3 } & 200 \\
\hline \text { Pop4 } & \\
\hline \text { Pop7 } & \\
\hline \text { Pop10 } & \\
\hline \text { Pop11 } & \\
\hline \text { Pop8 } & \\
\hline \text { Pop9 } &
\end{array}
$$
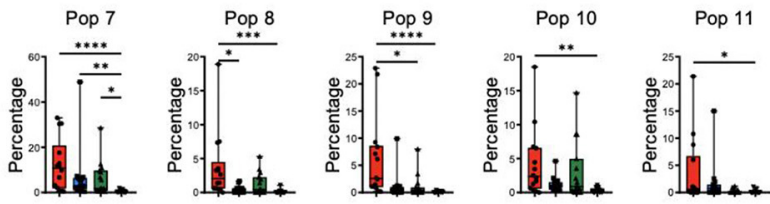

FIGURE 3 | CD8 T cell subsets, perforin expression and activated cells in COVID-19 patients. (A) Left: pseudocolor plots of concatenated peripheral CD8 T cells from healthy controls $(\mathrm{HC})$ and patients with mild, moderate and severe COVID-19. Four cell subsets were identified: naïve (CD27+CD45RA+), memory (CD27+CD45RA-), 
FIGURE 3 | effector-memory (CD27-CD45RA-), and terminal differentiated effector-memory (TEMRA) (CD27-CD45RA+). Numbers in the gates are the average of each subset. Right: boxplot graphs representation of the data. (B) Pseudocolor plots of concatenated peripheral CD8 T cells from HC and COVID-19 patients and boxplot graphs of the frequencies of activated naiive, memory, effector-memory and TEMRA cells. Numbers in the quadrants are the average of each subset. Activated T cells are identified by the coexpression of CD38 and HLA-DR. (C) Pseudocolor plots of concatenated peripheral CD8T cells and boxplot graphs showing the frequencies of PD-1+ naive, memory, effector-memory and TEMRA cells. Numbers in the gates are the average of PD-1+ cells in each subset. (D) Pseudocolor plots of concatenated peripheral CD8T cells and boxplot graphs of the frequencies of perforin positive naïve, memory, effector-memory and TEMRA cells. Numbers in the gates are the average of perforin positive cells in each subset. (E) tSNE projection of non-naive CD8 T cell populations (Pop) identified by FlowSOM clustering tool. (F) Fluorescence intensity of each Pop as indicated in the column-scaled z-score and boxplot graphs showing the frequencies of Pop7, Pop8, Pop9, Pop10, and Pop11 in HC and COVID-19 patients. Boxplots show the median and 25-75th percentiles, and the whiskers denote lowest and highest values. Each dot represents a donor. Significance of data in (A-D,G) was determined by the Kruskal-Wallis test followed by Dunn's multiple comparison test. ${ }^{*} p<0.05,{ }^{* \star} p<0.01,{ }^{\star \star \star} p<0.001$, and ${ }^{* \star * *} p<0.0001$.

Pop5 also belongs to the classical monocyte subset and is characterized by higher expression of HLA-DR, low CD300c and medium levels of CD300a and CD300e. In accordance with the results from conventional analysis, Pop 5 was most expanded in patients with mild and moderate disease, while the frequency of this population did not change in severe COVID-19 patients when compared with HC. Pop1, which is the predominant population in $\mathrm{HC}(\sim 50 \%$ of monocytes $)$ and is significantly reduced in patients, belongs to the classical monocyte subset and is characterized by high HLA-DR and CD300c expression, intermediate levels of CD300e and high levels of CD300a. Altogether, monocytes from COVID-19 patients have an activated phenotype, a gradual loss of HLA-DR expression that correlates with the severity of the disease and, very interestingly, a shift in the expression of CD300 molecules between moderate and severe disease.

\section{Perforin and Granzyme B Armed NK Cell Subsets Are Expanded in Patients With Severe Disease}

The role of NK cells in the recognition and elimination of virus-infected cells is well-documented, as well in modulating the adaptive immune response (48). But also, uncontrolled NK cell activation may contribute to hyper-inflammation and tissue injury (49). Therefore, we decided to perform a phenotypical analysis of NK cells from COVID-19 patients. Following a gating strategy previously described by us $(50,51)$ (Supplementary Figure 1), we first determined the frequency of the three circulating $\mathrm{NK}$ cell subsets, i.e., CD56 ${ }^{\text {bright }}, \mathrm{CD} 56^{\mathrm{dim}}$ and $\mathrm{CD} 56^{\text {neg }} \mathrm{NK}$ cells. CD56 $6^{\text {bright }}$ cells are considered a less mature subset than CD56 ${ }^{\mathrm{dim}} \mathrm{NK}$ cells (52). Our results showed that there were no significant differences in the frequencies of the three subsets between HC and COVID-19 patients (Figure 6A).

Next, we analyzed the expression of perforin and granzyme $\mathrm{B}$ in NK cells (Figure 6B). Results showed that both CD56 bright and CD $56^{\text {dim }}$ NK cells from COVID-19 patients exhibited higher levels of perforin and granzyme B as determined by the MFI of these markers. This increase in perforin and granzyme $B$ levels were associated with the severity of the disease (Figure 6B), suggesting that NK cells from patients with moderate and, even more with severe disease, have the potential to eliminate more efficiently target cells. As a subset, CD $56^{\mathrm{dim}} \mathrm{NK}$ cells continue to differentiate (52). During this process, among other phenotypical features, they lose the expression of NKG2A and sequentially acquire CD57 (52). Therefore, we determined the expression of
NKG2A and CD57 on CD56 ${ }^{\text {dim }}$ NK cells from HC and COVID19 patients to evaluate their maturation status. Results showed that there were no significant differences in the frequencies of the four subsets between HC and patients (Figure 6C). Nevertheless, the increased expression of perforin and granzyme $B$ that associated with the severity of the disease was also evident in each of the four subsets (Supplementary Figure 9A).

To gain more insight into the NK cell alterations observed in COVID-19, we used the FlowSOM clustering tool and compared the expression of the eight markers to define 16 populations (Figure 6D). Using this approach, we were able to identify some populations that were differentially expressed between COVID19 patients and HC (Figure 6E and Supplementary Figure 9B). Pop6, Pop7 and Pop11 represented CD56 ${ }^{\mathrm{dim}} \mathrm{NK}$ cells with relatively low amount of perforin and granzyme B expression. The frequency of these three populations was significantly higher in $\mathrm{HC}$, while patients with severe disease exhibited a lower frequency. Pop6 and Pop11 are CD57-, while Pop7 is CD57+. Related to the CD56 ${ }^{\text {bright }} \mathrm{NK}$ cell subset, both Pop14 and Pop15 were identified. While Pop14 does not express perforin or granzyme B, Pop 15 expresses high levels of these two cytolytic markers. As expected, the frequency of Pop14 was very low in patients with severe disease, while Pop15 was significantly expanded in severe COVID-19.

Despite the classification as innate cells, the discovery of memory properties in NK cells hints the role of this cell type in adaptive immunity and long-term responses $(48,53)$. In humans adaptive NK cells express NKG2C and lack signaling molecules such as $\mathrm{FcR} \gamma(\mathrm{Fc} \varepsilon \mathrm{RI} \gamma)$. It has been previously described that in patients with severe COVID-19 there is an increase in the frequency of adaptive NK cells in the circulation (30). Therefore, we studied this NK cell subset in our cohort of patients. First, we determined the frequencies of $\mathrm{NKG} 2 \mathrm{C}+, \mathrm{FcR} \gamma$ - and $\mathrm{CD} 57+\mathrm{NKG} 2 \mathrm{C}+$ subsets within the CD56 ${ }^{\text {dim }}$ NK cells (Figure 7A). Results showed that there were no significant differences in the frequency of these subsets, with the exception of an expansion of NKG2C+ and $\mathrm{CD} 57+\mathrm{NKG} 2 \mathrm{C}+$ cells from patients with moderate disease. When we simultaneously looked at the expression of the NKG2C and FcR $\gamma$ markers, we could distinguish four subsets: the NKG2C-FcR $\gamma+$ subset that represents the conventional $\mathrm{NK}$ cells, and the three different subsets of adaptive NK cells, i.e., NKG2C-FcR $\gamma^{-}, \mathrm{NKG} 2 \mathrm{C}+\mathrm{FcR} \gamma$-, and NKG2C+FcR $\gamma+$ (Figure 7B). The conventional NKG2C-FcR $\gamma+$ subset was the largest in both patients and $\mathrm{HC}$. The frequency of these four subsets was similar in patients and $\mathrm{HC}$ (Figure $7 \mathbf{B}$ and 
A
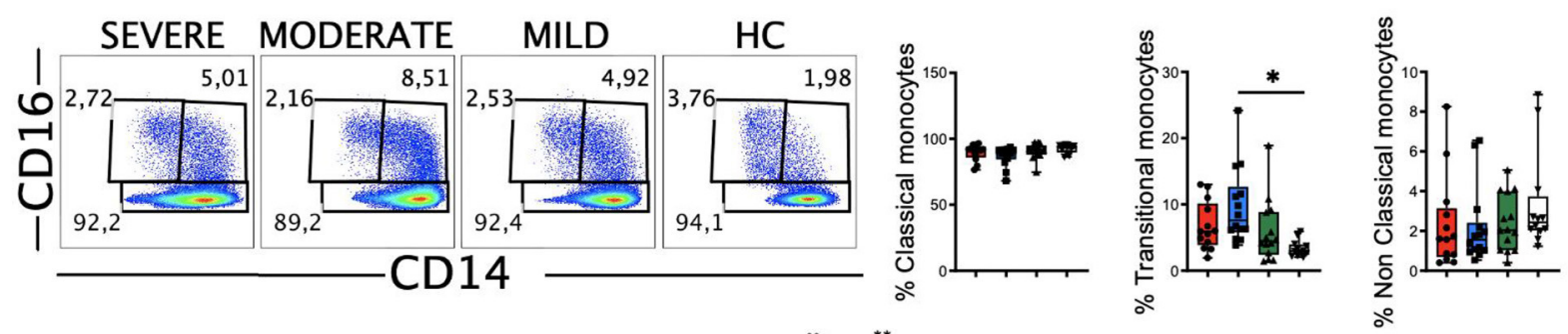

B

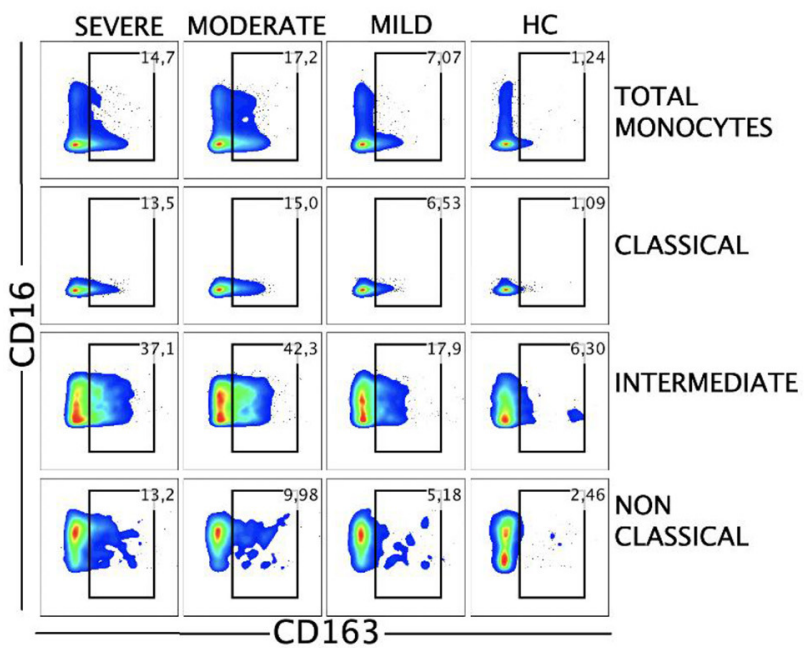

D
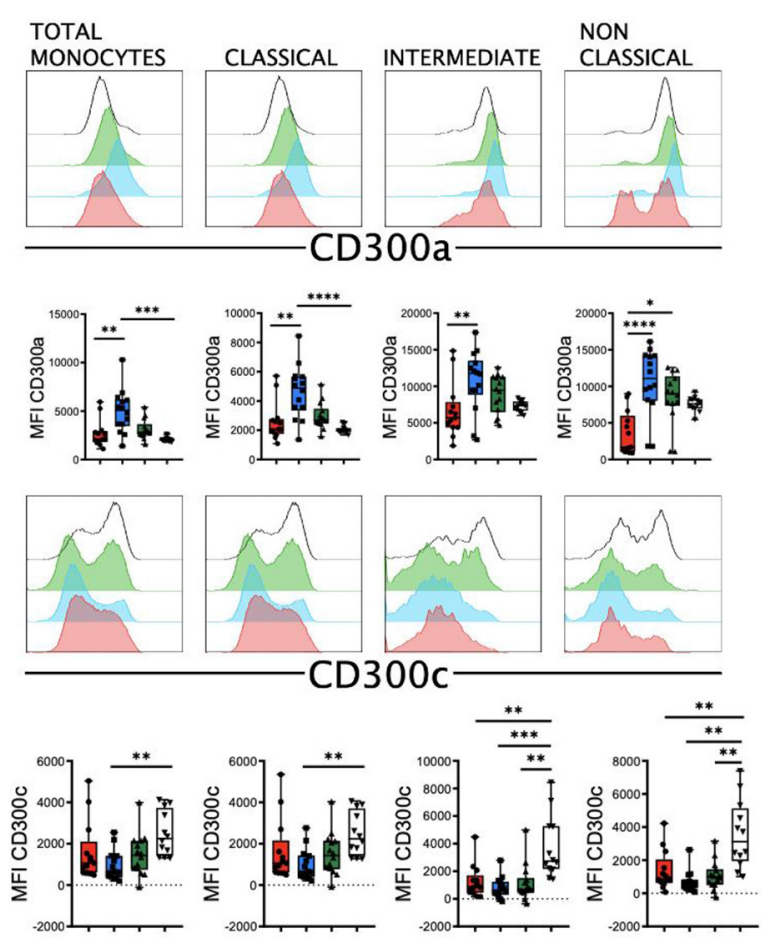

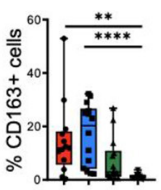

$\stackrel{0}{\overline{0}}^{60} \stackrel{* *}{* * *}$
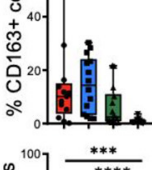

${ }_{0}^{100} \stackrel{* * *}{* * * *}$

C
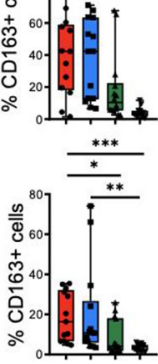

$\therefore$.

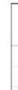

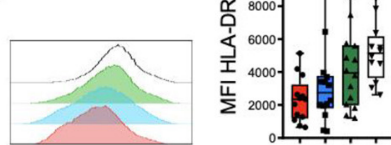

TOTAL MONOCYTES
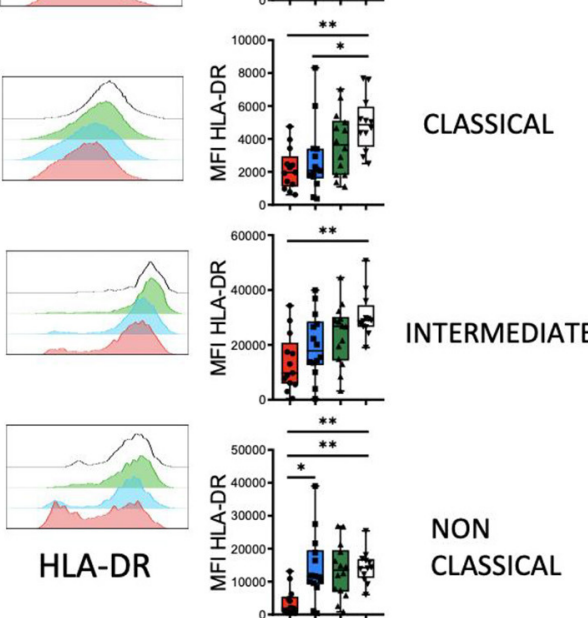

NON

CLASSICAL
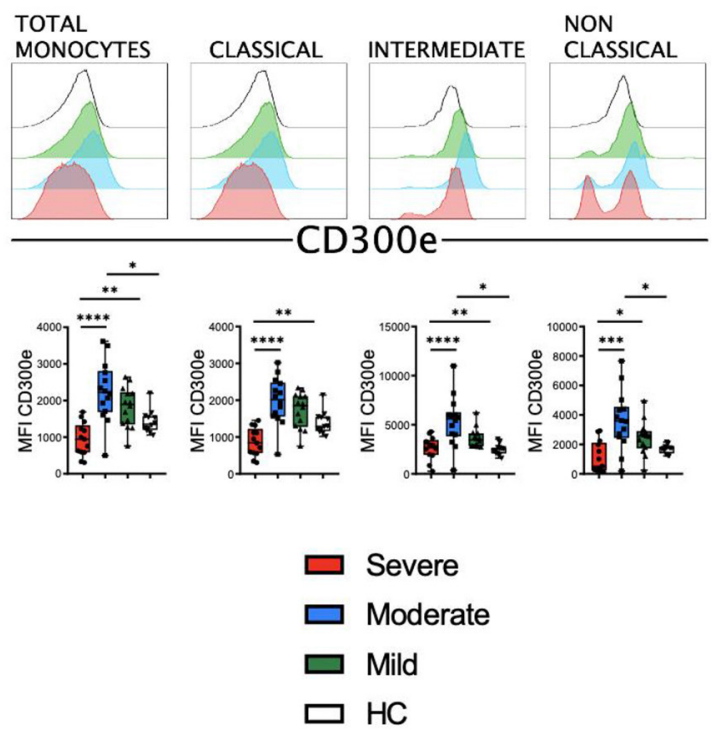

FIGURE 4 | CD163, HLA-DR and CD300 receptors expression in monocytes from COVID-19 patients. (A) Pseudocolor plots of concatenated monocytes cells from healthy controls $(\mathrm{HC})$ and patients and boxplot graphs of the frequencies of classical (CD14++CD16-), intermediate (CD14++CD16+), and non-classical 
FIGURE 4 | (CD14+CD16++) monocyte subsets. Numbers in the gates are the average of each subset. (B) Pseudocolor plots of all concatenated monocytes and subsets and boxplot graphs showing the frequencies of CD163+ cells. Numbers in the gates are the average of CD163+ cells in each subset. (C) Histograms of concatenated monocytes and boxplot graphs showing the median fluorescence intensity (MFI) of HLA-DR in all and each monocyte subset. (D) Histograms of concatenated monocytes and boxplot graphs of the MFI of CD300a, CD300c, and CD300e in all and each monocyte subset. Boxplots show the median and 25-75th percentiles, and the whiskers denote lowest and highest values. Each dot represents a donor. Significance of data was determined by the Kruskal-Wallis test followed by Dunn's multiple comparison test. ${ }^{\star} p<0.05,{ }^{\star \star} p<0.01,{ }^{\star \star \star} p<0.001$, and ${ }^{\star \star \star \star} p<0.0001$.

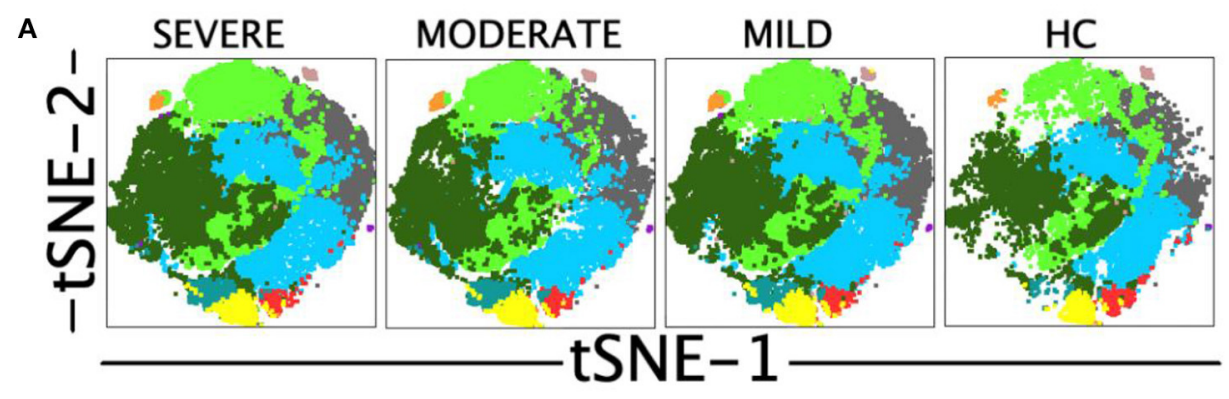

B
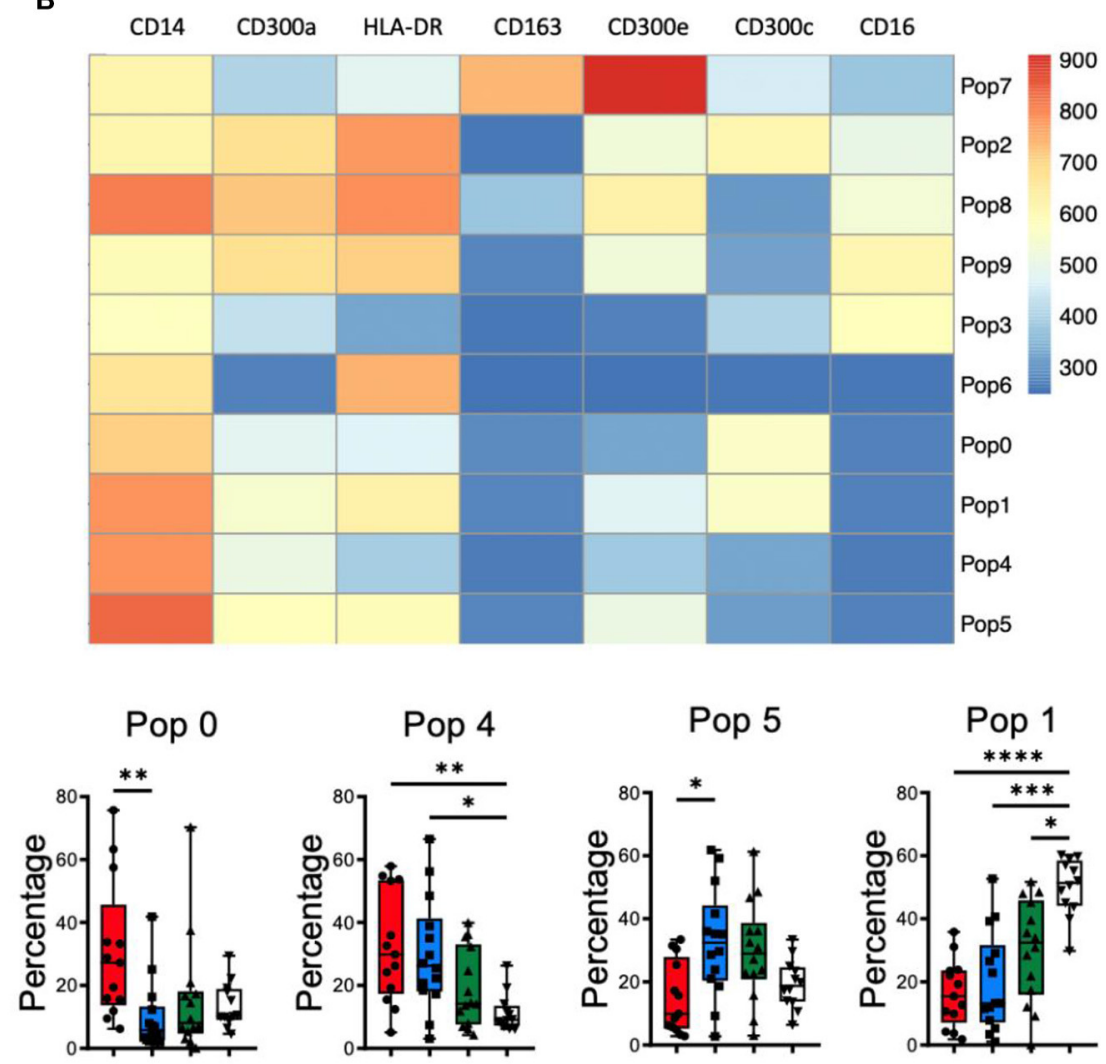

\begin{tabular}{|l|}
\hline Subset Name \\
\hline Pop9 \\
\hline Pop8 \\
\hline Pop7 \\
\hline Pop6 \\
\hline Pop5 \\
\hline Pop4 \\
\hline Pop3 \\
\hline Pop2 \\
\hline Pop1 \\
\hline Pop0 \\
\hline
\end{tabular}

FIGURE 5 | Unsupervised analysis of monocytes in COVID-19 patients. (A) tSNE projection of monocyte populations (Pop) identified by FlowSOM clustering tool. (B) Fluorescence intensity of each Pop as indicated in the column-scaled z-score and boxplot graphs showing the frequencies of Pop0, Pop1, Pop4, and Pop5 in healthy controls $(\mathrm{HC})$ and COVID-19 patients. Boxplots show the median and 25-75th percentiles, and the whiskers denote lowest and highest values. Each dot represents a donor. Significance of data was determined by the Kruskal-Wallis test followed by Dunn's multiple comparison test. ${ }^{*} p<0.05,{ }^{* *} p<0.01$, ${ }^{* \star *} p<0.001$, and ${ }^{* * \star *} p<$ 0.0001 .

Supplementary Figure 10A). Nevertheless, the expression of perforin and granzyme $\mathrm{B}$ was higher in each adaptive $\mathrm{NK}$ cell subset from COVID-19 patients when compared with HC (Supplementary Figure 10B).
It is well-known that human adaptive NK cells are expanded in individuals that are infected by $\operatorname{CMV}(48,54)$. Hence, we studied adaptive NK cells expansion according to the CMV serology status of patients and healthy controls. As reported by 
A
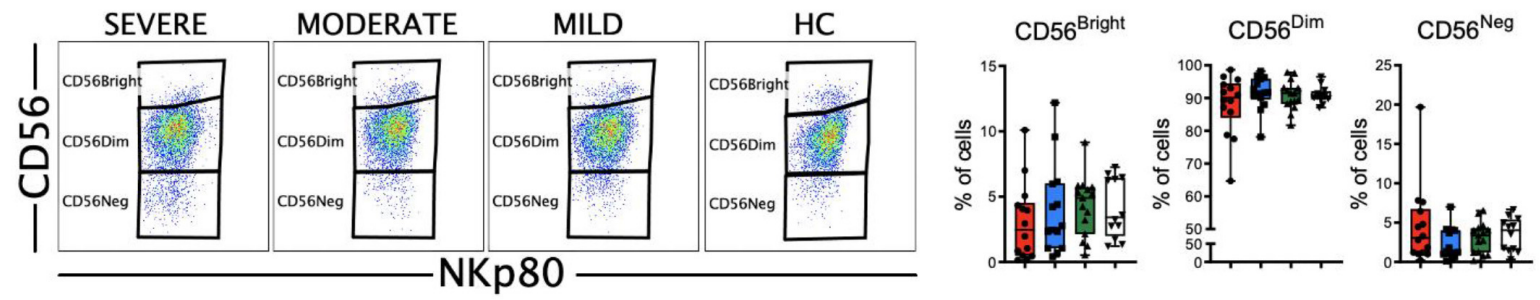

\section{CD56 ${ }^{\text {Bright }}$}
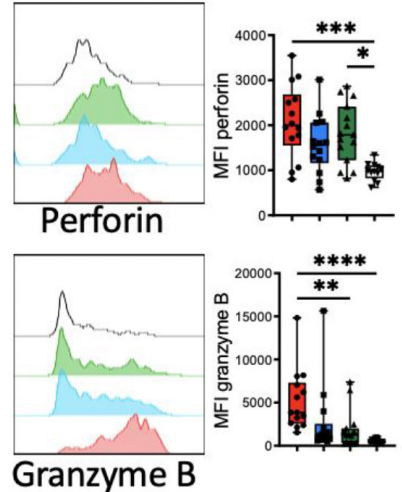

CD56 Dim

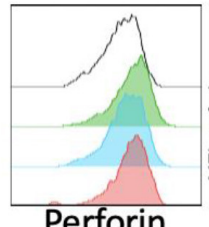

Perforin

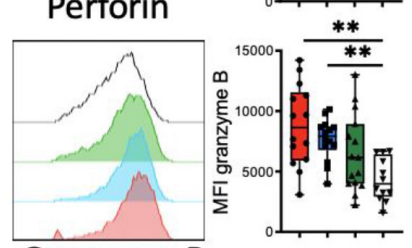

Granzyme B

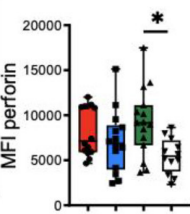

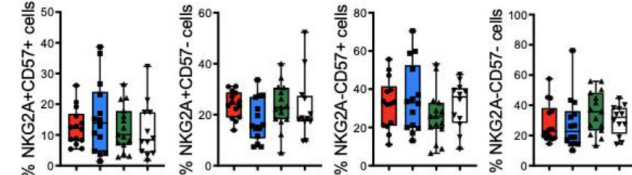

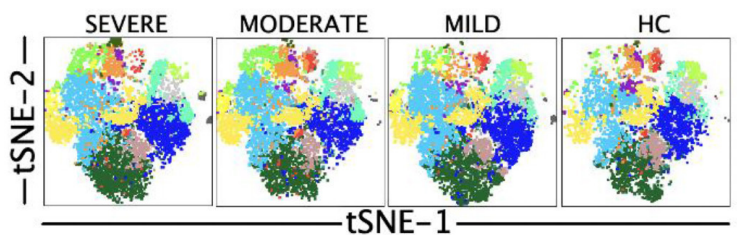

E NKG2A NKG2C FcRy CD56 CD57 CD16 Perforin GzmB
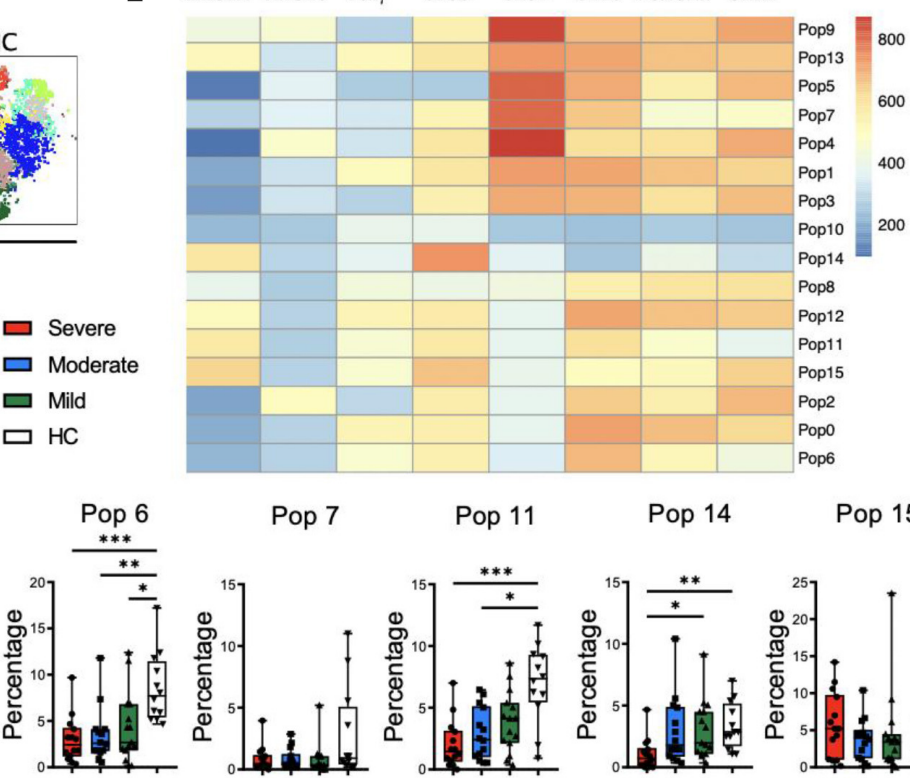

Pop 7

Pop 11

Pop 14

Pop 15

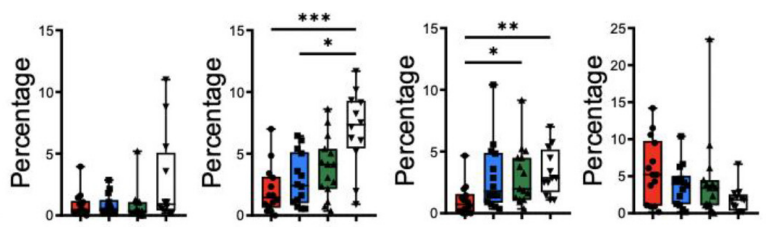

Pop7

Pop6

Pop5

Pop4

Pop3

Pop2

Pop1

- Pop0

FIGURE 6 | Perforin and granzyme B expression in NK cell subsets from COVID-19 patients. (A) Pseudocolor plots of concatenated NK cells from healthy controls $(\mathrm{HC})$ and patients and boxplot graphs of the frequencies of CD56 bright (CD56++NKp80+), CD56 dim (CD56+NKp80+) and CD56 ${ }^{\text {neg }}$ (CD56-NKp80+) NK cell subsets (B) Histograms of concatenated CD56 bright (left) and CD56 dim (right) NK cells and boxplot graphs of the median fluorescence intensity (MFI) of perforin (upper) and granzyme B (lower). (C) Pseudocolor plots of concatenated CD56 dim NK cells from HC and patients and boxplot graphs of the frequencies of the four subsets based in the expression of the CD57 and NKG2A differentiation markers. Numbers in the gates are the average of each subset. (D) tSNE projection of NK cells populations (Pop) identified by FlowSOM clustering. (E) Fluorescence intensity of each Pop as indicated in the column-scaled z-score and boxplot graphs showing the frequencies of Pop6, Pop7, Pop11, Pop14, and Pop15 in HC and COVID-19 patients. Boxplots show the median and 25-75 th percentiles, and the whiskers denote lowest and highest values. Each dot represents a donor. Significance of data in (A-C) was determined by the Kruskal-Wallis test followed by Dunn's multiple comparison test. ${ }^{\star} p<0.05,{ }^{* \star} p<0.01,{ }^{\star \star \star} p<0.001$, and ${ }^{\star \star \star \star} p<0.0001$ 
A
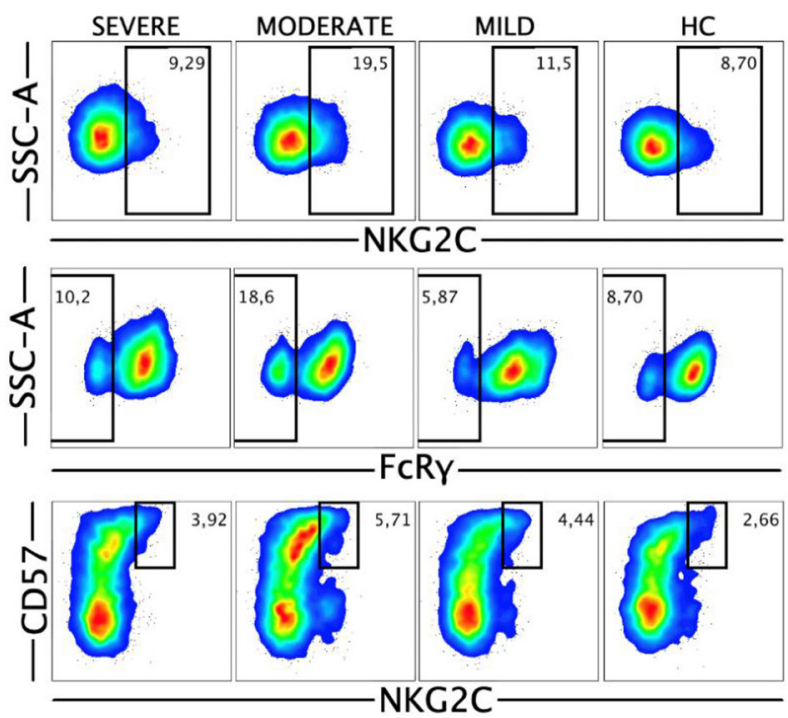
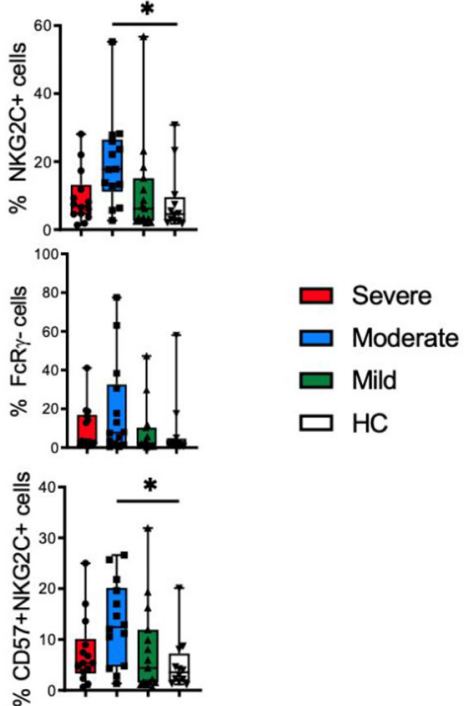

B

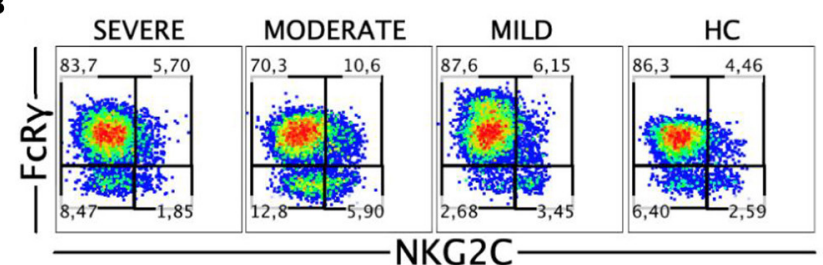

C

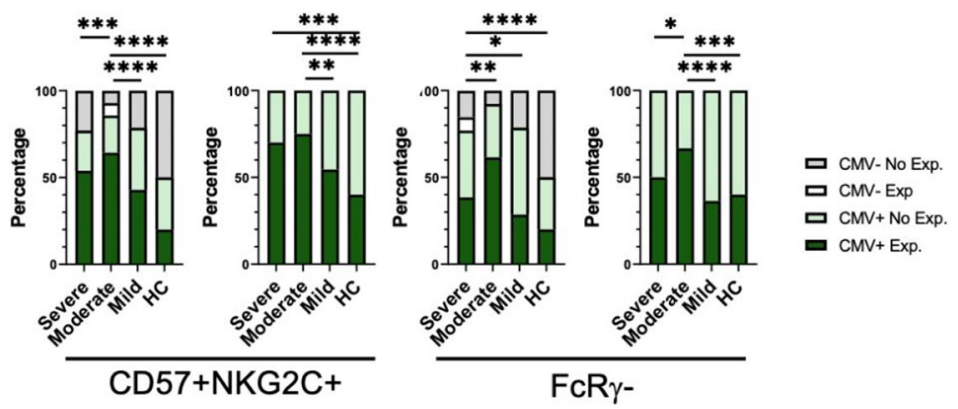

D

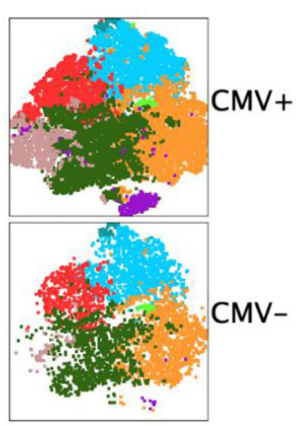

E

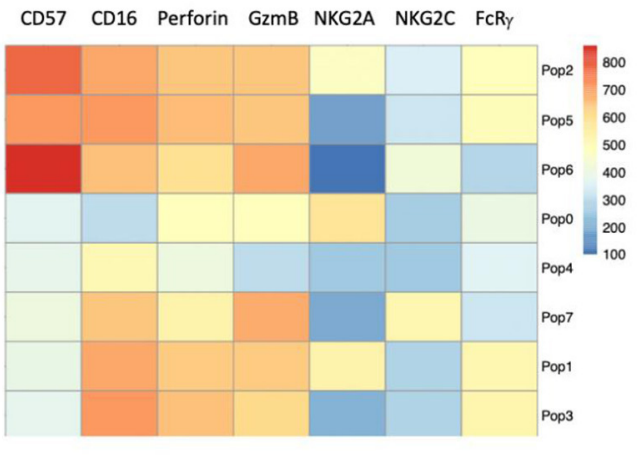

FIGURE 7 | Adaptive NK cells in COVID-19. (A) Pseudocolor plots of concatenated CD56 dim NK cells from healthy controls (HC) and patients and boxplot graphs of the frequencies of NKG2C+, FCR ${ }^{-}$, and CD57+NKG2C+ NK cell subsets. Numbers in the gates are the average of each subset. Boxplots show the median and 25-75th percentiles, and the whiskers denote lowest and highest values. Each dot represents a donor. Significance of data was determined by the Kruskal-Wallis test 
FIGURE 7 | followed by Dunn's multiple comparison test. ${ }^{*} p<0.05$. (B) Pseudocolor plots of concatenated CD56 dim NK cells from healthy controls (HC) and COVID-19 patients showing the expression of NKG2C and FcR $\gamma$. Numbers in the quadrants are the average of each subset. (C) Percentage of individuals from the indicated groups having or not having adaptive NK cell expansions. Significance of data was determined by chi-squared test. ${ }^{*} p<0.05$, ${ }^{* \star} p<0.01$, ${ }^{\star \star \star} p<0.001$, and ${ }^{\star \star \star \star} p<0.0001$. (D) tSNE projection of CD56 dim NK cells populations (Pop) identified by FlowSOM clustering tool from CMV + and CMV- donors. (E) Fluorescence intensity of each Pop as indicated in the column-scaled z-score and boxplot graphs showing the frequencies of Pop6 and Pop7 in CMV+ and CMV- HC and COVID-19 patients. Each dot represents a donor.

Maucourant et al. (30), we have determined that there is an expansion of adaptive CD57+NKG2C + NK cells when they are more than $5 \%$ of circulating CD56 ${ }^{\mathrm{dim}}$ cells. In addition, we have also determined that there is an expansion of adaptive FcR $\gamma$ - NK cells when they represent more than $7 \%$ of the CD56 ${ }^{\mathrm{dim}}$ cells. Results in Figure 7C shows that CMV-seronegative individuals do not have expansions of adaptive NK cells, except for one patient with moderate disease, in which the NKG2C+CD57+ cells represented more than 5\%, and another patient with severe disease, in which the FcR $\gamma$-cells were more than $7 \%$. When only the CMV-seropositive individuals were taken into account, we observed a significant expansion of adaptive NK cells in patients with moderate and severe COVID-19, which was more pronounced when $\mathrm{NKG} 2 \mathrm{C}+\mathrm{CD} 57+$ cells were taken into account instead of FcR $\gamma$-cells (Figure 7C).

To better understand the differences in NK cell subsets between $\mathrm{CMV}$-seropositive and CMV-seronegative individuals we used the FlowSOM clustering tool and compared the expression of seven markers to define 8 populations (Figure 7D). Using this approach, we were able to identify some populations that were differentially expressed between the two groups of donors (Figure 7E and Supplementary Figure 10C). Specifically, the adaptive NK cells Pop6 and Pop7 were characterized by the phenotype NKG2C+FcR $\gamma$-, and while Pop6 was CD57+, Pop7 was CD57-. As expected, the frequencies of Pop6 and Pop7 were higher in CMV-seropositive HC and COVID-19 patients, although we did not see significant differences.

\section{Statistical Analysis Reveals the Relationships Between Circulating T Cells, NK Cells and Monocytes With Disease Severity in COVID-19 Patients}

We first performed a bivariate analysis of 203 clinical laboratory and flow cytometry variables (Supplementary Table 4). We selected the statistically significant variables for a multivariate analysis. Then, to reduce the number of variables to include in the multivariate analysis we performed a principal component analysis (PCA) (Figure 8A). Components 1 to 4 explained around $73.7 \%$ of the variance, and components 1 and 2 explained around $60.8 \%$ of the variance (Supplementary Figure 11A). In Supplementary Figure 11B the contribution of each variable to components 1 to 4 is shown. The expression of CD300 molecules in monocytes and of granzyme B in CD56 bright $\mathrm{NK}$ cells are variables, along with the frequency of lymphocytes and neutrophils, that significantly contribute to component 1. For component 2, the CD300 receptors expression in monocytes, frequency of neutrophils and lymphocytes, along with CRP, fibrinogen, lymphocyte count and PD-1 in CD4 effector-memory
T cells, are variables that contribute significantly. Given that there are three categories (mild, moderate and severe) we performed multinomial logistic regression models. When we compared patients with a mild disease with those with a moderate or severe disease, results showed that component 1 is significantly different between patients with mild and severe disease, while component 2 is significantly different between patients with mild and moderate disease (Figure 8B, upper panel). On the other hand, when we compared patients with moderate disease with those with a mild and severe disease, we could see that component 1 was significantly different between patients with a moderate and severe disease and, as expected, component 2 was different between patients with moderate and mild disease (Figure 8B, lower panel).

Next, we performed correlation analysis. A different correlogram pattern was observed between $\mathrm{HC}$ and patients groups when we looked at the correlation between the significant flow cytometry variables (Supplementary Figure 12). Then, the analysis was performed to look for associations between the general laboratory values, clinical features and degrees of severity with the significant flow cytometry variables (Figure 8C). Indeed, the correlogram revealed many direct and inverse correlations providing a very valuable insight into the flow cytometry variables that were associated with the degree of severity. Very importantly, a strong direct association of activated $\mathrm{T}$ cells and granzyme B expression in CD56 ${ }^{\text {bright }} \mathrm{NK}$ cells with severe disease was observed. Furthermore, and as expected, there was a strong direct association between activated $\mathrm{T}$ cells and granzyme B expression in CD56 ${ }^{\text {bright }} \mathrm{NK}$ cells with clinical features of severity such as oxygen therapy, days with oxygen therapy, days in ICU, thrombosis/embolism and bilateral lung infiltrations. Related to clinical laboratory, we observed a direct correlation between activated T cells and granzyme B expression in CD56 ${ }^{\text {bright }} \mathrm{NK}$ cells with ferritin, counts and frequency of neutrophils, D-dimer, and CRP, and an inverse association with hemoglobin and counts and frequency of lymphocytes.

On the other hand, a very interesting picture emerged when the expression of CD300 receptors on monocytes was taken into consideration. A positive correlation between CD300a and CD300e expression with moderate disease was observed. However, a shift in their correlation was detected in patients with severe disease. In fact, an inverse correlation between CD300a and CD300e expression in monocytes with other clinical features of severity was also observed. We also looked at the expression of HLA-DR in monocytes, and there was also an inverse correlation with clinical features associated to severity such as days in ICU, dead, CRP, D-dimer, etc. Altogether, these results indicate that activated $\mathrm{T}$ cells, high expression of granzyme B in NK cells and a shift in the expression of 
A

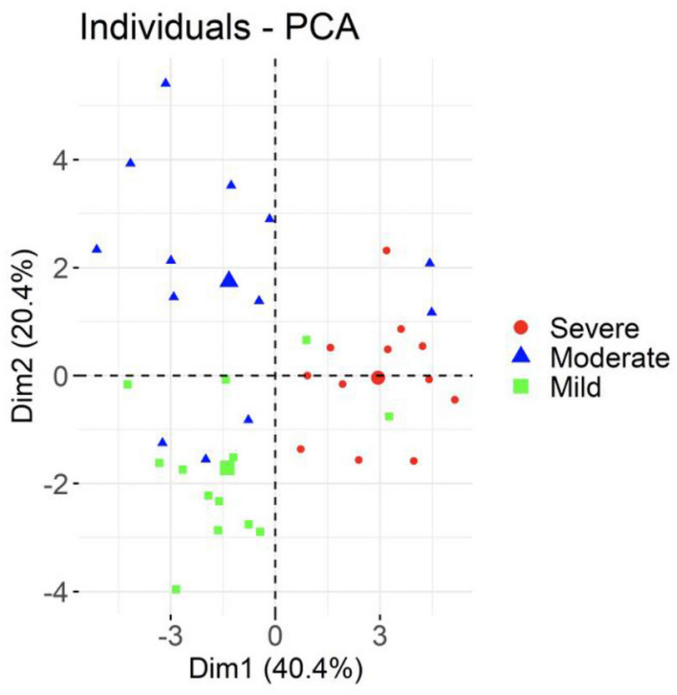

C

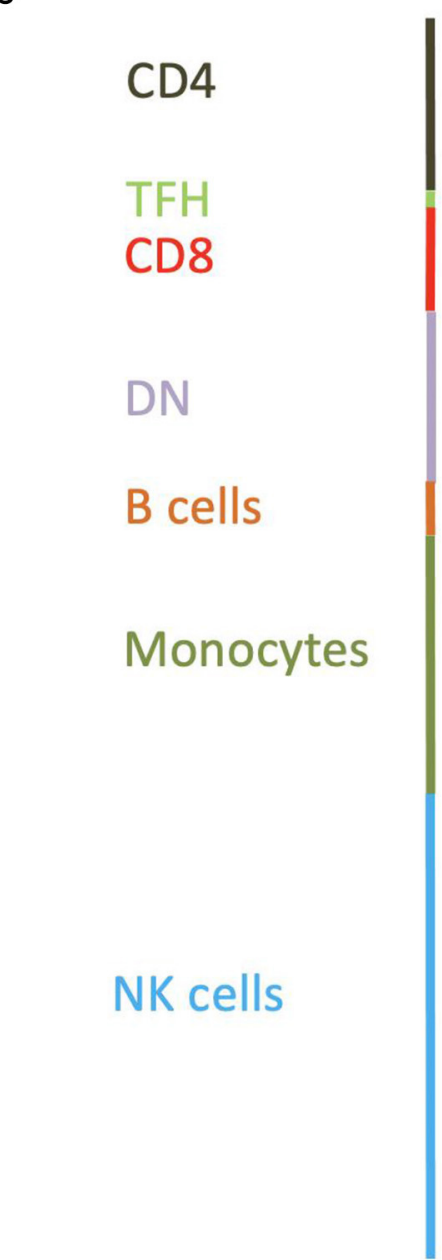

B

\begin{tabular}{|ccccc|} 
& $\begin{array}{c}\text { MODERATE } \\
\text { OR (95\% Cl) }\end{array}$ & p-value & $\begin{array}{c}\text { SEVERE } \\
\text { OR }(95 \% \text { CI) }\end{array}$ & p-value \\
\hline Comp1 & $0.98(0.6 ; 1.61)$ & 0.936 & $2.18(1.19 ; 3.99)$ & 0.011 \\
\hline Comp2 & $3.67(1.47 ; 9.18)$ & 0.005 & $1.44(0.53 ; 3.86)$ & 0.472 \\
\hline
\end{tabular}

\section{Moderate vs Mild and Severe}

\begin{tabular}{|ccrcr|} 
& $\begin{array}{c}\text { MILD } \\
\text { OR }(95 \% \text { Cl })\end{array}$ & p-value & $\begin{array}{c}\text { SEVERE } \\
\text { OR }(95 \% \text { Cl })\end{array}$ & p-value \\
\hline Comp1 & $1.02(0.62 ; 1.68)$ & 0.936 & $2.23(1.21 ; 4.11)$ & 0.010 \\
\hline Comp2 & $0.27(0.11 ; 0.68)$ & 0.005 & $0.39(0.15 ; 1.05)$ & 0.062 \\
\hline
\end{tabular}
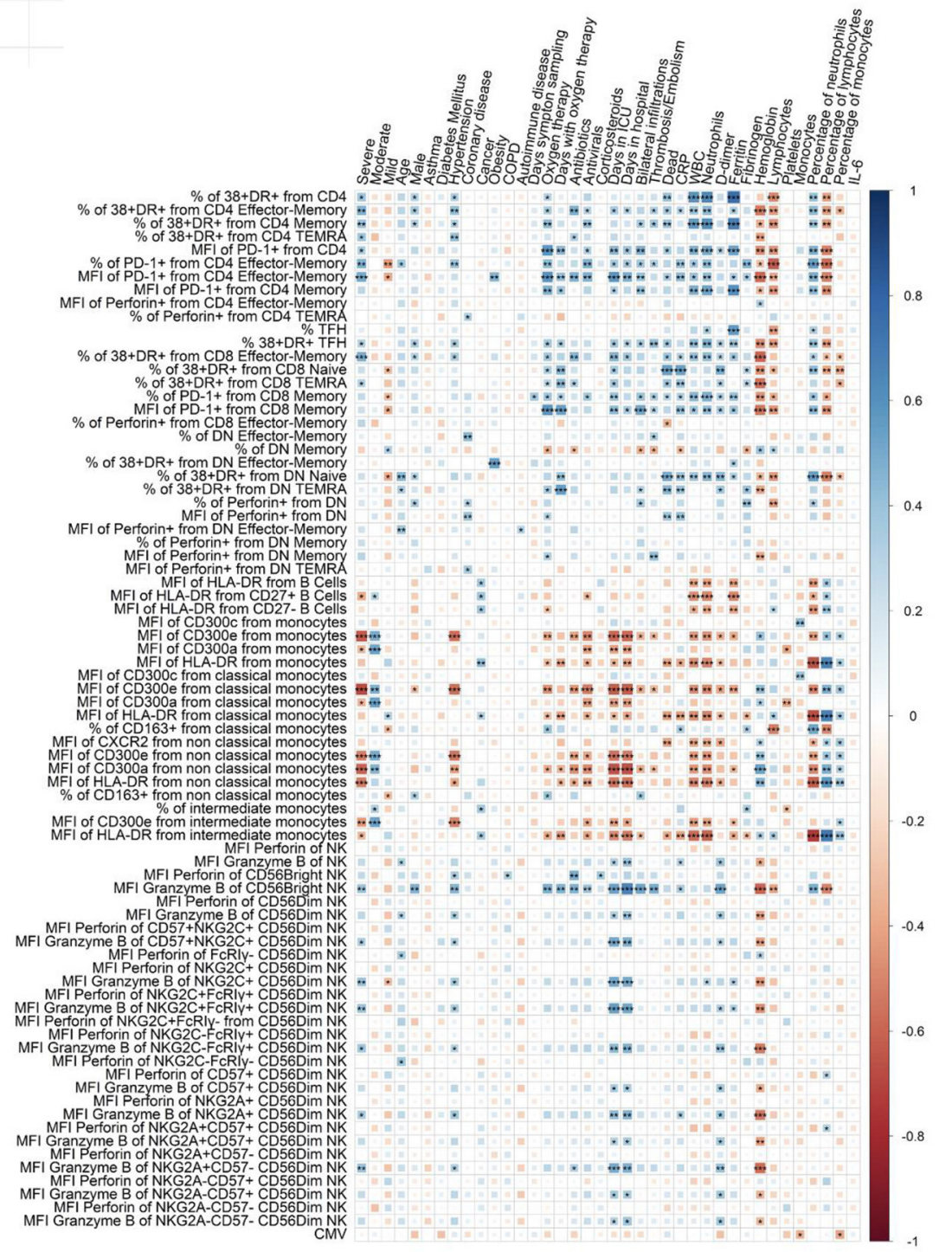

p-values: ${ }^{*}<0.05, * *<0.01,{ }^{* * *}<0.001$

FIGURE 8 | Multivariate analysis and correlation studies of immune cell phenotypes and clinical parameters. (A) Representation of the principal component analysis (PCA) results obtained with the most discriminant markers between patients groups. (B) Multinomial logistic regression model and statistical significance. Upper: 
FIGURE 8 | Patients with mild disease vs. patients with moderate and severe disease. Lower: Patients with moderate disease vs. patients with mild and severe disease. Odd ratio (OR), 95\% confidence interval (Cl) and p-values are indicated. (C) Correlogram showing Spearman correlation of the indicated flow cytometry data and clinical features for COVID-19 patients. Only flow cytometry data that were statistically significant from the bivariate analysis (Supplementary Table 4) were considered for the analysis.

CD300 receptors are very significant features of patients with severe COVID-19.

\section{DISCUSSION}

In this study, we have carried out a phenotypic characterization of circulating immune cells in order to determine correlates that may help to distinguish between degrees of severity in COVID-19. In addition, the findings that we have obtained could shed some light into the underlying mechanisms of the disease immunopathogenesis, especially in severe cases. Among other findings, an increased activation status of all $\mathrm{T}$ cell subsets and highly armed cytotoxic cells, mostly NK cells and TEMRA and effector-memory $\mathrm{T}$ cells, are characteristics of severe disease. Furthermore, to our knowledge, we have uncovered a previously unrecognized alteration and a shift in the pattern of expression of CD300 receptors on monocytes. Very importantly, conventional and unsupervised analyses lead to very similar conclusions highlighting the relevance of our findings.

A possible limitation of our study is the size of the cohort of patients with COVID-19 $(n=44)$ and HC $(n=12)$. However, and to avoid problems related to the diversity of participants, we have been very careful in choosing a cohort that is as uniform as possible before we performed the study.

It is widely accepted that immune dysregulation contributes to the pathology seen in severe cases of SARS-CoV-2 infection. A consistent finding in many studies, including ours, is that COVID-19 patients display robust activation of the T cell pool, although a considerable portion of patients have minimal levels of activation compared to HC (11). Our study shows that the frequency of activated $\mathrm{T}$ cells increases with the degree of severity of the disease. Furthermore, a very significant correlation was observed between many of the activated $\mathrm{T}$ cell subsets with severe disease while, as expected, an inverse association was observed with patients that experienced a mild disease. We also observed that $\mathrm{CD} 4$ and $\mathrm{CD} 8 \mathrm{~T}$ cells from patients with severe disease tended to have higher levels of perforin. How these activated and perforin containing $\mathrm{T}$ cells contribute to the disease pathology is not well-known. However, it has been proposed that highly differentiated $\mathrm{T}$ cells may induce damage during SARS-CoV-2 infection in a mechanism involving the induction of ligands, as for example MICA/B, for activating NK cell receptors in the respiratory epithelium. The recruited terminally differentiated $\mathrm{T}$ cells, which among other things are characterized by the expression of high levels of perforin and NK cell receptors, may recognize and induce $\mathrm{T}$ cell receptorindependent killing of epithelial cells in the respiratory tract and lungs (41). Therefore, it is tempting to speculate that in patients with severe COVID-19, the expansion of activated $\mathrm{T}$ cells, especially the effector-memory and TEMRA cells, have a role in the pathology of the disease. To confirm this affirmation, an in depth analysis of these cell subsets (in blood and tissues) from patients with different degrees of severity is required, including a complete study of their NK cell receptor repertoire. On the other hand, we observed that cytotoxic (perforin containing) CD4 T cells are expanded in a subset of COVID-19 patients. Both protective and pathogenic role for these cells during viral infection have been proposed $(39,40)$. Even more, it has been postulated that an expansion of cytotoxic $\mathrm{CD} 4 \mathrm{~T}$ cells drives cardiovascular disease in certain inflammatory conditions and they are triggered by CMV infection (40). There is no doubt that it is necessary to determine if these cytotoxic CD4 T cells have some role in tissue damage and thrombotic complications in COVID-19.

Although we did not find a significant increase in the frequency of circulating TFH, they were more activated in patients with severe COVID-19, suggesting that these CD38+HLA-DR+ TFH had a recent antigen encounter and may be providing B cell help (38), possibly as a part of an extrafollicular response, which somehow is explained by the observed low levels of CXCR5 in B cells from patients with severe disease. It has been shown in other viral infections that activated circulating TFH correlates with blood plasmablasts frequency (38). We did not observe such correlation and, furthermore, we only saw an increase in the plasmablasts frequency in patients with moderate disease. This is in contrast with other studies (11) and we do not know the reason for this discrepancy. Nevertheless, it is important to point out that we have not determined the specific SARS-CoV-2 plasmablast response, but the frequency of total plasmablasts.

Similar to Maucourant et al. (30), we have also found that COVID-19 patients exhibited expansions of adaptive NK cells as well as highly armed NK cells. Considering that adaptive NK cells are not a uniform NK cell subset (54), it is important to point out that we observed the expansions when we use the two more common gating strategies to identify adaptive NK cells: CD57+NKG2C+ cells and FcR $\gamma$ - cells. Expansions of adaptive NK cells occurred mostly, but not exclusively, on those individuals that were CMV-seropositive, raising the question of a possible CMV reactivation in COVID-19. However, the lack of proliferation of CMV specific T cells in SARS-CoV-2 infection and the absence of correlation between the expansions of adaptive NK cells with specific CMV IgG titers argues against the CMV reactivation as the cause $(30,55)$.

There was an apparent increase in the levels of perforin and granzyme B in NK cells from COVID-19 patients that correlated with the disease severity. This increased arming of NK cells was very evident in the CD56 $6^{\text {bright }}$ subset, which 
in patients with severe COVID-19 expressed high levels of perforin and even higher of granzyme B. Interestingly, two populations (Pop14 and Pop15) that differed in the expression of perforin, granzyme B and CD16 were identified within the CD56 $6^{\text {bright }}$ NK cells. Some authors have previously suggested that $\mathrm{CD} 56^{\text {bright }} \mathrm{CD} 16+\mathrm{NK}$ cells represent a more mature stage within the CD56 $6^{\text {bright }}$ subset (56). Therefore, we could conclude that in severe COVID-19 there is a shift toward more mature NK cells within the CD56 $6^{\text {bright }}$ cells. On the other hand, we did not observe a difference in the maturation status of the $\mathrm{CD} 56^{\mathrm{dim}}$ subset according to the expression of NKG2A and CD57.

Correlation studies showed a strong association of granzyme $\mathrm{B}$ expression in CD56right $\mathrm{NK}$ cells with severe disease as shown by clinical features such as bilateral infiltrations, thrombosis/embolism, oxygen therapy, neutrophilia, etc. A less strong association was also observed with granzyme $\mathrm{B}$ expression in CD56 ${ }^{\mathrm{dim}} \mathrm{NK}$ cells. The presence of these highly armed NK cells in patients with severe disease may suggest the possibility to eliminate more efficiently target cells, including virus-infected cells, and activated $\mathrm{T}$ cells that may cause immunopathology, and therefore modulate the adaptive immune response (57). But also, these NK cells can cause tissue damage in a way similar to how respiratory syncytial virus causes acute lung damage (49). Undoubtedly, more studies are required to know how these two aspects of NK cells contribute to the immunopathogenesis of COVID-19.

A decrease in non-classical monocytes and an increase in the intermediate subset have been associated to a severe and mild COVID-19, respectively (22-24). Although we did not find significant differences between patients and $\mathrm{HC}$ in the frequency of monocyte subsets, a tendency to a diminution in the frequency of the non-classical subset and an increase in the intermediate monocytes were evident. It is possible that our results are due to the panel we have used for the identification of monocytes (Supplementary Figure 1). Although we have identified monocytes as CD14+HLA$\mathrm{DR}+\mathrm{CD} 66 \mathrm{~b}-$ cells, including the CD14 $4^{\text {low }}$ HLA-DR+CD66bcells, still it is possible that the comprehensive identification of the three monocyte subsets is not complete. Future studies must include a panel including antibodies for specific markers to exclude T cells, B cells and NK cells. It is also very important to exclude basophils (FceRla+HLA-DR-), plasma cells (CD45RA $\left.{ }^{\text {high }} \mathrm{CD} 38^{\text {high }}\right)$ and $\mathrm{CD} 88$-CD116- cells from the population of interest. Then, dendritic cells (DCs) subsets and monocytes should be separately analyzed, for which markers such as CD88, CD89, FceR1a, CD1c, CD123, etc. would be very helpful, even for differentiating monocytes from the CD14+CD163+ DC3 subset (35). On the other hand, and in agreement with previous results $(10,23,24)$, we observed a decrease in HLA-DR expression in monocytes. The decrease was gradual and it was very significant in patients with severe COVID-19. HLA-DR ${ }^{\text {low }}$ monocytes are considered dysfunctional and are an established surrogate marker of immunosuppression in sepsis (58). Acute infections trigger an emergency myelopoiesis that is characterized by the mobilization of immature myeloid cells, which are linked to immunosuppressive functions (59). Therefore, it is reasonable to propose that an increment in dysfunctional monocytes and an emergency myelopoiesis are factors that contribute to the development of severe disease.

The CD300 molecules are type I transmembrane proteins expressed on the surface of immune cells and are divided in two groups: activating and inhibitory receptors (44). CD300 receptors are able to bind different ligands, mostly lipids such as phosphatidylserine, phosphatidylethanolamine and ceramide $(44,45,60,61)$. They regulate many signaling pathways, as for example monocyte and neutrophil activation (44-46, 62, 63). The importance of CD300 molecules in several pathological conditions has been highlighted by multiple studies describing the role of this family of receptors in allergic disorders, autoimmune and inflammatory diseases, cancer, sepsis and viral infections $(44,45,47)$. In this study we have observed a very intriguing expression pattern of the CD300 molecules in COVID19 patients. The expression of the inhibitory CD300a receptor and of the activating CD300e receptor gradually increased in monocytes from patients with mild and moderate disease. It is well-known that activation of neutrophils and monocytes with pro-inflammatory stimuli, such as LPS and GM-CSF, increased the expression of CD300a and CD300e $(46,63)$. Therefore, it is plausible to assume that in patients with moderate and mild disease the increased expression in these two CD300 receptors is a consequence of the inflammatory milieu. However, a change in the pattern of CD300a and CD300e receptors expression happened in patients with severe COVID-19 that exhibited very low levels of these two molecules. The cause for this shift is not known. However, some clues could be found in the HL-60 acute myeloid leukemia cell model to study the differentiation toward monocytes and neutrophils (63). Undifferentiated HL-60 cell do not express CD300 molecules, but when neutrophil and monocyte differentiation was induced, a significant expression of CD300a was observed, indicating that the CD300a receptor expression is developmentally regulated (63). Therefore, a possible explanation for the decrease in CD300a expression is that the circulating monocytes and granulocytes are more immature (and possibly more dysfunctional) that those observed in mild and moderate COVID-19. In the same way, we have found that HL-60 cells do not express CD300e and that their differentiation toward monocytes and granulocytes barely induces its expression (data not shown), also suggesting that immature cells do not express CD300e. This immature phenotype is also supported by the lower HLA-DR expression in monocytes from patients with severe disease. It is interesting to see how the expression levels of an inhibitory receptor $(\mathrm{CD} 300 \mathrm{a})$ and activating receptor (CD300e) are regulated in the same manner in COVID-19 patients. It could be because these molecules share, at least partially, similar mechanisms regulating their expression, something that is supported by the increased expression of both receptors following LPS stimulation (46). On the other hand, the functional significance of the altered expression of $\mathrm{CD} 300$ receptors is unknown, but one can speculate that the functional status of monocytes and their responses to soluble and externalized lipids will be 
significantly affected. For example, the release from infected cells of phosphatidylserine-expressing particles directly into the blood stream (64) could interact with CD300 receptors expressed on monocytes and granulocytes, which will respond in a different manner depending on the expression levels of those receptors. Evidently, more studies are required to understand not only the exact mechanisms involved in the regulation of CD300 receptors expression in COVID-19, but also the role that they play in this disease. Besides that, the determination of CD300 receptors expression in COVID-19 is very important as the statistical, including PCA analysis, and correlation studies show.

In conclusion, the two important findings of this study were that the unsupervised analysis obtained similar results and reached similar conclusions than the conventional analysis, and that the statistical and correlation studies corroborated that several immune alterations were in close relationship with the severity of the disease as shown by the clinical features and clinical laboratory data. This study could be improved in the future by increasing the number of recruited patients, performing longitudinal studies, analyzing in more depth immune cell subsets, as for example terminal differentiated $\mathrm{T}$ cells and monocytes, from blood and tissues, obtaining more comprehensive clinical data, etc. Still, our study, along with those published by others, provides a compilation of immune responses data that could provide additional light on the immune mechanisms behind the development of severe COVID-19.

\section{DATA AVAILABILITY STATEMENT}

The original contributions presented in the study are included in the article/Supplementary Material, further inquiries can be directed to the corresponding author/s.

\section{ETHICS STATEMENT}

The studies involving human participants were reviewed and approved by Basque Ethics Committee for Research with Medicines (CEIm-E). The patients/participants provided their written informed consent to participate in this study.

\section{REFERENCES}

1. Huang C, Wang Y, Li X, Ren L, Zhao J, Hu Y, et al. Clinical features of patients infected with 2019 novel coronavirus in Wuhan, China. Lancet. (2020) 395:497-506. doi: 10.1016/S0140-6736(20)30183-5

2. Zhou F, Yu T, Du R, Fan G, Liu Y, Liu Z, et al. Clinical course and risk factors for mortality of adult inpatients with COVID-19 in Wuhan, China: a retrospective cohort study. Lancet. (2020) 395:105462. doi: 10.1016/S0140-6736(20)30566-3

3. Klok FA, Kruip MJHA, van der Meer NJM, Arbous MS, Gommers DAMPJ, Kant KM, et al. Incidence of thrombotic complications in critically ill ICU patients with COVID-19. Thromb Res. (2020) 191:1457. doi: 10.1016/j.thromres.2020.04.013

4. Lodigiani C, Iapichino G, Carenzo L, Cecconi M, Ferrazzi P, Sebastian T, et al. Venous and arterial thromboembolic complications in COVID-19

\section{AUTHOR CONTRIBUTIONS}

$\mathrm{FB}$ conceived the project. $\mathrm{OZ}$ and $\mathrm{FB}$ designed experiments and obtained samples from healthy controls. IS-B, JN-A, NI-A, and EA-A obtained the clinical samples and clinical data from COVID-19 patients. RP-G determined IL-6 and CMV serology from patients and healthy controls. OZ stained and acquired flow cytometry samples. GA-P and FB performed flow cytometry analysis. GA-P and SP-F performed computational and statistical analysis. GA-P, SP-F, and FB compiled figures. OZ, GA-P, IT, and $\mathrm{AO}$ provided intellectual input. FB wrote the manuscript. All authors reviewed the manuscript.

\section{FUNDING}

This work is supported by a grant from the Agencia Estatal de Investigación Project PID2019-109583RBI00/AEI/10.13039/501100011033 and a grant from the Department of Health, Basque Government (2020111045). $\mathrm{OZ}$ is recipient of a postdoctoral contract funded by Instituto de Salud Carlos III-Contratos Sara Borrell 2017 (CD17/0128) and the European Social Fund (ESF)-The ESF invests in your future. GA-P is recipient of a fellowship from the BBK Fundazioa (1543/2006_0001) and from the Jesús de Gangoiti Barrera Foundation (FJGB20/002). IT is recipient of a predoctoral contract funded by the Department of Education, Basque Government (PRE_2020_2_007). AO is recipient of a fellowship from the Jesús de Gangoiti Barrera Foundation (FJGB20/007). $\mathrm{FB}$ is an Ikerbasque Research Professor, Ikerbasque, Basque Foundation for Science.

\section{ACKNOWLEDGMENTS}

We thank all patients and healthy controls who participated in this study and the staff from the Basque Biobank for Research. This manuscript has been released as a pre-print at bioRxiv (65).

\section{SUPPLEMENTARY MATERIAL}

The Supplementary Material for this article can be found online at: https://www.frontiersin.org/articles/10.3389/fimmu. 2021.655934/full\#supplementary-material

patients admitted to an academic hospital in Milan, Italy. Thromb Res. (2020) 191:9-14. doi: 10.1016/j.thromres.2020.04.024

5. Tang N, Li D, Wang X, Sun Z. Abnormal coagulation parameters are associated with poor prognosis in patients with novel coronavirus pneumonia. J Thromb Haemost. (2020) 18:844-7. doi: 10.1111/jth.14768

6. Rapkiewicz A V., Mai X, Carsons SE, Pittaluga S, Kleiner DE, Berger JS, et al. Megakaryocytes and platelet-fibrin thrombi characterize multi-organ thrombosis at autopsy in COVID-19: a case series. EClinicalMedicine. (2020) 24:100434. doi: 10.1016/j.eclinm.2020.100434

7. Vabret N, Britton GJ, Gruber C, Hegde S, Kim J, Kuksin M, et al. Immunology of COVID-19: current state of the science. Immunity. (2020) 52:91041. doi: 10.1016/j.immuni.2020.05.002

8. Blanco-Melo D, Nilsson-Payant BE, Liu W-C, Uhl S, Hoagland D, Møller $\mathrm{R}$, et al. Imbalanced host response to SARS-CoV-2 drives development of COVID-19. Cell. (2020) 181:1036-45.e9. doi: 10.1016/j.cell.2020.04.026 
9. Moore JB, June CH. Cytokine release syndrome in severe COVID-19. Science. (2020) 368:473-4. doi: 10.1126/science.abb8925

10. Giamarellos-Bourboulis EJ, Netea MG, Rovina N, Akinosoglou K, Antoniadou A, Antonakos N, et al. Complex immune dysregulation in COVID-19 patients with severe respiratory failure. Cell Host Microbe. (2020) 27:992-1000.e3. doi: 10.1016/j.chom.2020.04.009

11. Mathew D, Giles JR, Baxter AE, Oldridge DA, Greenplate AR, Wu JE, et al. Deep immune profiling of COVID-19 patients reveals distinct immunotypes with therapeutic implications. Science. (2020) 369:eabc8511. doi: 10.1126/science.abc8511

12. Kuri-Cervantes L, Pampena MB, Meng W, Rosenfeld AM, Ittner CAG, Weisman AR, et al. Comprehensive mapping of immune perturbations associated with severe COVID-19. Sci Immunol. (2020) 5:eabd7114. doi: 10.1126/sciimmunol.abd7114

13. Laing AG, Lorenc A, del Molino del Barrio I, Das A, Fish M, Monin L, et al. A dynamic COVID-19 immune signature includes associations with poor prognosis. Nat Med. (2020) 26:1623-35. doi: 10.1038/s41591-020-1038-6

14. Su Y, Chen D, Yuan D, Lausted C, Choi J, Dai CL, et al. Multi-omics resolves a sharp disease-state shift between mild and moderate COVID-19. Cell. (2020) 183:1479-95.e20. doi: 10.1016/j.cell.2020.10.037

15. Zhou R, To KK-W, Wong Y-C, Liu L, Zhou B, Li X, et al. Acute SARSCoV-2 infection impairs dendritic cell and T cell responses. Immunity. (2020) 53:864-77.e5. doi: 10.1016/j.immuni.2020.07.026

16. Del Valle DM, Kim-Schulze S, Huang H-H, Beckmann ND, Nirenberg S, Wang B, et al. An inflammatory cytokine signature predicts COVID-19 severity and survival. Nat Med. (2020) 26:1636-43. doi: 10.1038/s41591-020-1051-9

17. Herold T, Jurinovic V, Arnreich C, Lipworth BJ, Hellmuth JC, von BergweltBaildon M, et al. Elevated levels of IL- 6 and CRP predict the need for mechanical ventilation in COVID-19. J Allergy Clin Immunol. (2020) 146:12836.e4. doi: $10.1016 /$ j.jaci.2020.05.008

18. Yang Y, Shen C, Li J, Yuan J, Wei J, Huang F, et al. Plasma IP-10 and MCP-3 levels are highly associated with disease severity and predict the progression of COVID-19. J Allergy Clin Immunol. (2020) 146:119127.e4. doi: 10.1016/j.jaci.2020.04.027

19. Hadjadj J, Yatim N, Barnabei L, Corneau A, Boussier J, Smith N, et al. Impaired type I interferon activity and inflammatory responses in severe COVID-19 patients. Science. (2020) 369:718-24. doi: 10.1126/science.abc6027

20. Carissimo G, Xu W, Kwok I, Abdad MY, Chan Y-H, Fong S-W, et al. Whole blood immunophenotyping uncovers immature neutrophil-to-VD2 T-cell ratio as an early marker for severe COVID-19. Nat Commun. (2020) 11:5243. doi: 10.1038/s41467-020-19080-6

21. Wilk AJ, Rustagi A, Zhao NQ, Roque J, Martínez-Colón GJ, McKechnie JL, et al. A single-cell atlas of the peripheral immune response in patients with severe COVID-19. Nat Med. (2020) 26:1070-1076. doi: 10.1038/s41591-020-0944-y

22. Sánchez-Cerrillo I, Landete P, Aldave B, Sánchez-Alonso S, Sánchez-Azofra A, Marcos-Jiménez A, et al. COVID-19 severity associates with pulmonary redistribution of $\mathrm{CD} 1 \mathrm{c}+\mathrm{DCs}$ and inflammatory transitional and nonclassical monocytes. J Clin Invest. (2020) 130:6290-300. doi: 10.1172/JCI140335

23. Schulte-Schrepping J, Reusch N, Paclik D, Baßler K, Schlickeiser S, Zhang B, et al. Severe COVID-19 is marked by a dysregulated myeloid cell compartment. Cell. (2020) 182:1419-40.e23. doi: 10.1016/j.cell.2020.08.001

24. Silvin A, Chapuis N, Dunsmore G, Goubet A-G, Dubuisson A, Derosa L, et al. Elevated calprotectin and abnormal myeloid cell subsets discriminate severe from mild COVID-19. Cell. (2020) 182:1401-18.e18. doi: 10.1016/j.cell.2020.08.002

25. Li M, Guo W, Dong Y, Wang X, Dai D, Liu X, et al. Elevated exhaustion levels of $\mathrm{NK}$ and $\mathrm{CD} 8+\mathrm{T}$ cells as indicators for progression and prognosis of COVID-19 disease. Front Immunol. (2020) 11:580237. doi: 10.3389/fimmu.2020.580237

26. Zheng M, Gao Y, Wang G, Song G, Liu S, Sun D, et al. Functional exhaustion of antiviral lymphocytes in COVID-19 patients. Cell Mol Immunol. (2020) 17:533-5. doi: 10.1038/s41423-020-0402-2

27. Mazzoni A, Salvati L, Maggi L, Capone M, Vanni A, Spinicci M, et al. Impaired immune cell cytotoxicity in severe COVID-19 is IL-6 dependent. J Clin Invest. (2020) 130:4694-703. doi: 10.1172/JCI138554
28. Osman M, Faridi RM, Sligl W, Shabani-Rad M-T, Dharmani-Khan P, Parker A, et al. Impaired natural killer cell counts and cytolytic activity in patients with severe COVID-19. Blood Adv. (2020) 4:50359. doi: 10.1182/bloodadvances.2020002650

29. Demaria O, Carvelli J, Batista L, Thibult M-L, Morel A, André P, et al. Identification of druggable inhibitory immune checkpoints on natural killer cells in COVID-19. Cell Mol Immunol. (2020) 17:9957. doi: $10.1038 / \mathrm{s} 41423-020-0493-9$

30. Maucourant C, Filipovic I, Ponzetta A, Aleman S, Cornillet M, Hertwig L, et al. Natural killer cell immunotypes related to COVID-19 disease severity. Sci Immunol. (2020) 5:eabd6832. doi: 10.1126/sciimmunol.abd6832

31. Jiang $\mathrm{Y}$, Wei $\mathrm{X}$, Guan J, Qin S, Wang Z, Lu H, et al. COVID19 pneumonia: CD8+ T and NK cells are decreased in number but compensatory increased in cytotoxic potential. Clin Immunol. (2020) 218:108516. doi: 10.1016/j.clim.2020.108516

32. Stegelmeier AA, van Vloten JP, Mould RC, Klafuric EM, Minott JA, Wootton SK, et al. Myeloid cells during viral infections and inflammation. Viruses. (2019) 11:168. doi: 10.3390/v11020168

33. Mann ER, Menon M, Knight SB, Konkel JE, Jagger C, Shaw $\mathrm{TN}$, et al. Longitudinal immune profiling reveals key myeloid signatures associated with COVID-19. Sci Immunol. (2020) 5:eabd6197. doi: 10.1126/sciimmunol.abd6197

34. Gatti A, Radrizzani D, Viganò P, Mazzone A, Brando B. Decrease of nonclassical and intermediate monocyte subsets in severe acute SARS-CoV-2 infection. Cytom Part A. (2020) 97:887-90. doi: 10.1002/cyto.a.24188

35. Kvedaraite E, Hertwig L, Sinha I, Ponzetta A, Hed Myrberg I, Lourda $\mathrm{M}$, et al. Major alterations in the mononuclear phagocyte landscape associated with COVID-19 severity. Proc Natl Acad Sci USA. (2021) 118:e2018587118. doi: 10.1073/pnas.2018587118

36. Vitte J, Diallo AB, Boumaza A, Lopez A, Michel M, Allardet-Servent J, et al. A granulocytic signature identifies COVID-19 and its severity. J Infect Dis. (2020) 222:1985-96. doi: 10.1093/infdis/jiaa591

37. Schönrich G, Raftery MJ. The PD-1/PD-L1 axis and virus infections: a delicate balance. Front Cell Infect Microbiol. (2019) 9:207. doi: $10.3389 /$ fcimb.2019.00207

38. Crotty S. T follicular helper cell biology: a decade of discovery and diseases. Immunity. (2019) 50:1132-48. doi: 10.1016/j.immuni.2019.04.011

39. Sanchez-Martinez A, Perdomo-Celis F, Acevedo-Saenz L, Rugeles MT, Velilla PA. Cytotoxic CD4+ T-cells during HIV infection: targets or weapons? J Clin Virol. (2019) 119:17-23. doi: 10.1016/j.jcv.2019.08.004

40. Broadley I, Pera A, Morrow G, Davies KA, Kern F. Expansions of cytotoxic CD4+CD28- $\mathrm{T}$ cells drive excess cardiovascular mortality in rheumatoid arthritis and other chronic inflammatory conditions and are triggered by CMV infection. Front Immunol. (2017) 8:195. doi: 10.3389/fimmu.2017.00195

41. Akbar AN, Gilroy DW. Aging immunity may exacerbate COVID-19. Science. (2020) 369:256-7. doi: 10.1126/science.abb0762

42. Halle S, Halle O, Förster R. Mechanisms and dynamics of $\mathrm{T}$ cell-mediated cytotoxicity in vivo. Trends Immunol. (2017) 38:432-43. doi: 10.1016/j.it.2017.04.002

43. Tippett E, Cheng W-J, Westhorpe C, Cameron PU, Brew BJ, Lewin SR, et al. Differential expression of CD163 on monocyte subsets in healthy and HIV-1 infected individuals. PLOS ONE. (2011) 6:e19968. doi: 10.1371/journal.pone.0019968

44. Borrego F. The CD300 molecules: an emerging family of regulators of the immune system. Blood. (2013) 121:195160. doi: 10.1182/blood-2012-09-435057

45. Zenarruzabeitia O, Vitallé J, Eguizabal C, Simhadri VR, Borrego F. The biology and disease relevance of $\mathrm{CD} 300 \mathrm{a}$, an inhibitory receptor for phosphatidylserine and phosphatidylethanolamine. J Immunol. (2015) 194:5053-60. doi: 10.4049/jimmunol.1500304

46. Zenarruzabeitia O, Vitallé J, García-Obregón S, Astigarraga I, Eguizabal C, Santos S, et al. The expression and function of human CD300 receptors on blood circulating mononuclear cells are distinct in neonates and adults. Sci Rep. (2016) 6:32693. doi: 10.1038/srep32693

47. Vitallé J, Terrén I, Orrantia A, Zenarruzabeitia O, Borrego F. CD300 receptor family in viral infections. Eur J Immunol. (2019) 49:36474. doi: 10.1002/eji.201847951 
48. Lam VC, Lanier LL. NK cells in host responses to viral infections. Curr Opin Immunol. (2017) 44:43-51. doi: 10.1016/j.coi.2016.11.003

49. Li F, Zhu H, Sun R, Wei H, Tian Z. Natural killer cells are involved in acute lung immune injury caused by respiratory syncytial virus infection. J Virol. (2012) 86:2251-8. doi: 10.1128/JVI.06209-11

50. Orrantia A, Terrén I, Izquierdo-Lafuente A, Alonso-Cabrera JA, Sandá V, Vitallé J, et al. A NKp80-based identification strategy reveals that CD56neg NK cells are not completely dysfunctional in health and disease. iScience. (2020) 23:101298. doi: 10.1016/j.isci.2020.101298

51. Orrantia A, Terrén I, Vitallé J, Astarloa-Pando G, Zenarruzabeitia O, Borrego F. Identification and functional analysis of human CD56neg NK cells by flow cytometry. STAR Protoc. (2020) 1:100149. doi: 10.1016/j.xpro.2020.100149

52. Di Vito C, Mikulak J, Mavilio D. On the way to become a natural killer cell. Front Immunol. (2019) 10:1812. doi: 10.3389/fimmu.2019.01812

53. Cerwenka A, Lanier LL. Natural killer cell memory in infection, inflammation and cancer. Nat Rev Immunol. (2016) 16:112-23. doi: 10.1038/nri.2015.9

54. Rölle A, Brodin P. Immune adaptation to environmental influence: the case of NK cells and HCMV. Trends Immunol. (2016) 37:23343. doi: 10.1016/j.it.2016.01.005

55. Sekine T, Perez-Potti A, Rivera-Ballesteros O, Strålin K, Gorin $\mathrm{J}-\mathrm{B}$, Olsson $\mathrm{A}$, et al. Robust $\mathrm{T}$ cell immunity in convalescent individuals with asymptomatic or mild COVID-19. Cell. (2020) 183:158-68.e14. doi: 10.1016/j.cell.2020.08.017

56. Campos C, López N, Pera A, Gordillo JJ, Hassouneh F, Tarazona R, et al. Expression of NKp30, NKp46 and DNAM-1 activating receptors on resting and IL-2 activated NK cells from healthy donors according to CMV-serostatus and age. Biogerontology. (2015) 16:671-83. doi: 10.1007/s10522-015-9581-0

57. Waggoner SN, Cornberg M, Selin LK, Welsh RM. Natural killer cells act as rheostats modulating antiviral T cells. Nature. (2012) 481:3948. doi: 10.1038/nature10624

58. Venet F, Demaret J, Gossez M, Monneret G. Myeloid cells in sepsis-acquired immunodeficiency. Ann N Y Acad Sci. (2020). doi: 10.1111/nyas.14333. [Epub ahead of print].

59. Loftus TJ, Mohr AM, Moldawer LL. Dysregulated myelopoiesis and hematopoietic function following acute physiologic insult. Curr Opin Hematol. (2018) 25:37-43. doi: 10.1097/MOH.0000000000000395
60. Izawa $\mathrm{K}$, Isobe $\mathrm{M}$, Matsukawa $\mathrm{T}$, Ito $\mathrm{S}$, Maehara $\mathrm{A}$, Takahashi $\mathrm{M}$, et al. Sphingomyelin and ceramide are physiological ligands for human LMIR3/CD300f, inhibiting FceRI-mediated mast cell activation. J Allergy Clin Immunol. (2014) 133:270-3.e1-7. doi: 10.1016/j.jaci.2013.08.008

61. Simhadri VR, Andersen JF, Calvo E, Choi S-C, Coligan JE, Borrego F. Human CD300a binds to phosphatidylethanolamine and phosphatidylserine, and modulates the phagocytosis of dead cells. Blood. (2012) 119:2799809. doi: 10.1182/blood-2011-08-372425

62. Simhadri VR, Mariano JL, Gil-Krzewska A, Zhou Q, Borrego F. CD300c is an activating receptor expressed on human monocytes. J Innate Immun. (2013) 5:389-400. doi: 10.1159/000350523

63. Alvarez Y, Tang X, Coligan JE, Borrego F. The CD300a (IRp60) inhibitory receptor is rapidly up-regulated on human neutrophils in response to inflammatory stimuli and modulates CD32a (Fc $\gamma$ RIIa) mediated signaling. Mol Immunol. (2008) 45:253-8. doi: 10.1016/j.molimm.2007.05.006

64. Lind SE. Phosphatidylserine is an overlooked mediator of COVID-19 thromboinflammation. Heliyon. (2021) 7:e06033. doi: 10.1016/j.heliyon.2021.e06033

65. Zenarruzabeitia O, Astarloa-Pando G, Terrén I, Orrantia A, Pérez-Garay $\mathrm{R}$, Seijas-Betolaza I, et al. $\mathrm{T}$ cell activation, highly armed cytotoxic cells and a sharp shift in monocytes CD300 receptors expression is characteristic of patients with severe COVID-19. bioRxiv [preprint]. (2020). doi: $10.1101 / 2020.12 .22 .423917$

Conflict of Interest: The authors declare that the research was conducted in the absence of any commercial or financial relationships that could be construed as a potential conflict of interest.

Copyright (C) 2021 Zenarruzabeitia, Astarloa-Pando, Terrén, Orrantia, PérezGaray, Seijas-Betolaza, Nieto-Arana, Imaz-Ayo, Pérez-Fernández, Arana-Arri and Borrego. This is an open-access article distributed under the terms of the Creative Commons Attribution License (CC BY). The use, distribution or reproduction in other forums is permitted, provided the original author(s) and the copyright owner(s) are credited and that the original publication in this journal is cited, in accordance with accepted academic practice. No use, distribution or reproduction is permitted which does not comply with these terms. 